\title{
A sliding observer for nonlinear process control
}

\author{
Gow-Bin Wang, Sheng-Shiou Peng and Hsiao-Ping Huang* \\ Department of Chemical Engineering, National Taiwan University, Taipei, Taiwan, 10617, \\ R.O.C.
}

(Received 18 December 1995; in revised form 5 August 1996; accepted 12 September 1996)

\begin{abstract}
Sliding observers are considered as nonlinear state estimators with good robustness to bounded modeling errors. In this paper we have developed sliding observers for process control. The obscrver is hence designed so as to possess invariant dynamic modes which can be assigned independently to achieve the desired performance. Convergence of the estimating algorithm is formulated by using Lyapunov stability theorems. Conditions for robustness to modeling errors are derived by analyzing the norms of estimation errors. For process control, servo-tracking and disturbance rejection for chemical reactors have been discussed by making use of this sliding observer. Simulation examples to demonstrate the construction and performance of this proposed sliding observer for chemical process control are also presented. (C) 1997 Elsevier Science Ltd. All rights reserved
\end{abstract}

Keywords: Sliding mode; observer; observability; state estimation; nonlinear system.

\section{INTRODUCTION}

Various methods for designing nonlinear controllers have been reported in the literature. Unless they are using ad hoc designs, most of the nonlinear controllers such as those using feedback linearization (Hunt et al., 1983; Kravaris and Chung, 1987), GMC (Lee and Sullivan, 1988) and many others require the feedback of state variables to implement the control strategies. In practice, however, complete state feedback is impractical in most applications. Although direct integration, i.c. open-loop observer, can be used to cstimate the required state feedback and works well for a few applications, a closed-loop observer with feedback correction is still desirable, especially, when the system consists of modeling error(s) or pure integrator(s).

Although the theories and applications for linear systems are well developed, development of observers for nonlinear systems still provides an open area for research. Till now, development of observers for nonlinear systems has encountered many difficulties, such as: requirement of extensive computational efforts, coupling with controllers, uncertainty in the performance or robustness, restrictive conditions to be satisfied, etc. Some of the major difficulties encountered in the development of observers, which are reported in literature, have been reviewed by Misawa and Hedrick (1989).

\footnotetext{
${ }^{*}$ Corresponding author.
}

In the last decade, attention has been focused on applying the transformed canonical forms for design (Bestle and Zeitz, 1983; Keller, 1987; Kantor, 1989; Ding et al., 1990, etc.). For most of the nonlinear systems, such a transformation can be defined through Lie derivatives of the output which is a function of state variables (Gibon-Fargeot et al., 1994; Alvarez-Ramírez, 1995). However, the resulting canonical forms are not strictly linear and the transformations thus involve exogenous inputs and a finite number of their derivatives. In case of simple systems, such difficulties involved in the transformations could be easily resolved. However, for systems having dimensions greater than two, solution of the coordinate transformations and existence of such transformations appear to be the bottleneck.

Recently, another type of observers that also use sliding mode have been reported. In the early work of Slotine et al. (1987), the observer was constructed for a second-order nonlinear dynamic system involving only single measurement. The extensions of such observers to $n$ th-order and multi-output systems have also been addressed in the literature. Further development in this field of sliding observer was made by Misawa (1988). Canudas and Slotine (1991) have further applied such observers in robot manipulators. In all the studies mentioned above, a framework similar to a Luenberger observer was used by appending a switching function with constant gains as part of feedback corrections. To obtain such gains, different procedures have been proposed (Misawa and Hedrick, 1989). These procedures require certain 
conditions to be satisfied or optimized with singular values which depend on the scaling factors. Thus, the procedures developed for determining the switching gains are complicated and the performance issue of the observers is also not addressed.

It is the purpose of the present paper to design sliding observers for control of nonlinear chemical processes. In chemical process control, measurements of indirect outputs are usually used to infer the key outputs which are rather difficult to be measured on-line. The sliding observer presented here is used to force these indirect but measurable outputs to lie on specified sliding surfaces, so that the resulting estimated states can be used to implement the nonlinear feedback control more efficiently. In order to design the observer independently, time-varying gains for the switching functions are used to keep the dynamic modes of these estimated states invariant regardless of the position of the states. For formulating the nominal convergence of the estimating algorithm, Lyapunov stability analysis has been used. Conditions for its robust stability are also derived herein. To demonstrate the potential use of this sliding observer, rejection of unknown disturbance by using a feedforwardlike control is illustrated. Similar use for the modelbased predictive control may be investigated in future research. Simulations for the application of chemical reactor control are given as illustrations for the potential uses of such sliding observers.

\section{MATHEMATICAL FORMULATION}

Consider a general nonlinear system as

$$
\begin{aligned}
& \dot{\mathbf{x}}=\mathbf{f}(\mathbf{x}, \mathbf{u}) \\
& \mathbf{y}=\mathbf{h}(\mathbf{x})
\end{aligned}
$$

where $\mathbf{x} \in \mathbf{R}^{n}$ is a vector of state variables, $\mathbf{u} \in \mathbf{R}^{m}$ is the input vector and $\mathbf{y} \in \mathbf{R}^{p}$ is the output vector.

To construct the nonlinear observers for the system given in eq. (1), it is essential to devise a correcting function $\Phi$ such that, integration of the following equation would produce estimates of $\mathbf{x}$, i.e. $\hat{\mathbf{x}}$ :

$$
\begin{aligned}
& \dot{\hat{\mathbf{x}}}=\mathbf{f}(\hat{\mathbf{x}}, \mathbf{u})+\boldsymbol{\Phi}(\mathbf{y}-\hat{\mathbf{y}}) \\
& \hat{\mathbf{y}}=\mathbf{h}(\hat{\mathbf{x}}) .
\end{aligned}
$$

As has been mentioned earlier, many nonlinear observers have been reported in the literature. Although not exhaustive, various methods of approach along with their disadvantages for the few reported nonlinear observers are summarized in Table 1. To overcome some major obstacles in the construction of nonlinear observer for process control, a sliding observer is presented here.

We will assume that the Jacobian matrix of $h$, i.e. $J(\mathbf{h}(x))$, exists and is of full rank for all $\mathbf{x} \in \mathbf{X}$, so that eq. (1) can now be transformed into the following system:

$$
\begin{aligned}
& \dot{\mathbf{z}}=\mathbf{f}^{*}(\mathbf{z}, \mathbf{u}) \\
& \mathbf{y}=\mathbf{C z}
\end{aligned}
$$

where $\mathbf{C}=\left[\begin{array}{ll}\mathbf{I}_{p} & \mathbf{0}\end{array}\right]$ and $\mathbf{X}$ is an open subset of $\mathbf{R}^{n}$.
Using eq. (3), $\mathbf{z}$ is partitioned into

$$
\mathbf{z}=\left[\begin{array}{l}
\mathbf{z}^{a} \\
\mathbf{z}^{b}
\end{array}\right] \text { with } \quad \mathbf{z}^{a}=\mathbf{y}
$$

so that an observer of the following form is constructed:

$$
\dot{\hat{\mathbf{z}}}=\mathbf{f}^{*}(\hat{\mathbf{z}}, \mathbf{u})+\mathbf{K}(t) \boldsymbol{\sigma}
$$

where $\mathbf{K}$ is a time-varying gain matrix and $\boldsymbol{\sigma}$ is given as follows:

$$
\boldsymbol{\sigma}=\left[\begin{array}{c}
\operatorname{sgn}\left(y_{1}-\hat{z}_{1}\right) \\
\operatorname{sgn}\left(y_{2}-\hat{z}_{2}\right) \\
\vdots \\
\operatorname{sgn}\left(y_{p}-\hat{z}_{p}\right)
\end{array}\right]
$$

and the sign function, $\operatorname{sgn}(t)$, is defined as

$$
\operatorname{sgn}(t)=\left\{\begin{aligned}
1 & \text { if } t>0 \\
-1 & \text { if } t<0 .
\end{aligned}\right.
$$

Further, let $\tilde{\mathbf{z}}^{a}=\mathbf{z}^{a}-\hat{\mathbf{z}}^{a}$ lie on sliding surfaces by applying sliding conditions. The switching gain matrix, $\mathbf{K}$, is formulated in order to keep the dynamic poles of $\tilde{\mathbf{z}}^{b}=\mathbf{z}^{b}-\hat{\mathbf{z}}^{b}$ invariant at desired constant values, which would lead to a good performance. Convergence of the estimating algorithm is formulated by using Lyapunov stability theorems and the robustness to modeling errors are derived via analysis of norms. Incorporation of such a sliding observer into a closed loop for process control is addressed in the following.

\section{SLIDING OBSERVER FOR SISO SYSTEMS}

In the following text, for convenience, we shall use $\mathbf{x}$, instead of $\mathbf{z}$, to denote the state in eq. (3). Consider a SISO nonlinear system of the following form:

$$
\begin{aligned}
& \dot{\mathbf{x}}=\mathbf{f}(\mathbf{x}, u) \\
& y=x_{1}
\end{aligned}
$$

where $\mathbf{x}=\left[\begin{array}{llll}x_{1} & x_{2} & \cdots & x_{n}\end{array}\right]^{\mathrm{T}}$ is a state vector and $u$ is an external input. The function $\mathbf{f}(\mathbf{x}, u)$ in eq. (6) is subject to a norm-bounded modeling error.

The sliding observer for this SISO system becomes

$$
\begin{gathered}
\dot{\hat{x}}_{1}=f_{1}(\hat{\mathbf{x}}, u)+k_{1} \operatorname{sgn}\left(x_{1}-\hat{x}_{1}\right) \\
\dot{\hat{x}}_{2}=f_{2}(\hat{\mathbf{x}}, u)+k_{2} \operatorname{sgn}\left(x_{1}-\hat{x}_{1}\right) \\
\vdots \\
\dot{\hat{x}}_{n}=f_{n}(\hat{\mathbf{x}}, u)+k_{n} \operatorname{sgn}\left(x_{1}-\hat{x}_{1}\right) .
\end{gathered}
$$

Let $\tilde{x}_{i}=x_{i}-\hat{x}_{i}, i=1,2, \cdots, n$; then the above equation becomes

$$
\begin{gathered}
\tilde{\tilde{x}}_{1}=\Delta f_{1}+\delta f_{1}-k_{1} \operatorname{sgn}\left(\tilde{x}_{1}\right) \\
\dot{\tilde{x}}_{2}=\Delta f_{2}+\delta f_{2}-k_{2} \operatorname{sgn}\left(\tilde{x}_{1}\right) \\
\quad \vdots \\
\dot{\tilde{x}}_{n}=\Delta f_{n}+\delta f_{n}-k_{n} \operatorname{sgn}\left(\tilde{x}_{1}\right)
\end{gathered}
$$


where $\Delta f_{i}=f_{i}(\mathbf{x}, u)-f_{i}(\hat{\mathbf{x}}, u)$ and $\delta f_{i}$ is the modeling error due to structural deviation.

We can define a sliding function in terms of $\tilde{x}_{1}$ as

$$
s=\tilde{x}_{1}
$$

and a sliding condition for $\tilde{x}_{1}$ as either

$$
\frac{1}{2} \frac{\mathrm{d}}{\mathrm{d} t} s^{2} \leqslant-\eta|s|
$$

or

$$
\frac{\mathrm{d}}{\mathrm{d} t} s=\quad \eta \operatorname{sgn}(s)
$$

where $\eta$ is a positive constant. In order to drive $\tilde{x}_{1}$ to its sliding surface, $\mathrm{d} \tilde{x}_{1} / \mathrm{d} t$ should have a sign opposite to that of $\tilde{x}_{1}$. To do so, we can assign the value to $k_{1}$ as

$$
k_{1} \geqslant \eta+F \text {. }
$$

Here, we assume that the dynamic uncertainty is explicitly bounded, i.e.

$$
\left|\Delta f_{1}+\delta f_{1}\right| \leqslant F
$$

where $F$ is a positive constant. Thus, inequality (12) can make the variant trajectories point towards the surface $s(t)=0$, where the tracking estimation error $\tilde{x}_{1}$ is zero.

Applying the concept of equivalent dynamics in accordance with Filippov (Slotine and Li, 1991), the convex combination of the dynamics on both sides of the surface $s(t)$ leads to

$$
\begin{aligned}
\dot{\tilde{x}}_{1} & =\gamma\left(\Delta f_{1}+\delta f_{1}+k_{1}\right)+(1-\gamma)\left(\Delta f_{1}+\delta f_{1}-k_{1}\right) \\
\dot{\tilde{x}}_{2} & =\gamma\left(\Delta f_{2}+\delta f_{2}+k_{2}\right)+(1-\gamma)\left(\Delta f_{2}+\delta f_{2}-k_{2}\right) \\
& \vdots \\
\dot{\bar{x}}_{n} & =\gamma\left(\Delta f_{n}+\delta f_{n}+k_{n}\right)+(1-\gamma)\left(\Delta f_{n}+\delta f_{n}-k_{n}\right) .
\end{aligned}
$$

When $k_{1}$ is determined from eq. (12), we have $\dot{s}=\dot{\tilde{x}}_{1}=0$, i.e.

$$
\gamma\left(\Delta f_{1}+\delta f_{1}+k_{1}\right)+(1-\gamma)\left(\Delta f_{1}+\delta f_{1}-k_{1}\right)=0 .
$$

Hence, the value of $\gamma$ is given as

$$
\gamma=\frac{k_{1}-\Delta f_{1}-\delta f_{1}}{2 k_{1}}
$$

Substituting eq. (15) into eq. (14) gives the reducedorder sliding observer dynamics in the form of

$$
\begin{aligned}
\dot{\tilde{x}}_{2} & =\Delta f_{2}+\delta f_{2}-\left(k_{2} / k_{1}\right)\left(\Delta f_{1}+\delta f_{1}\right) \\
\dot{\tilde{x}}_{3} & =\Delta f_{3}+\delta f_{3}-\left(k_{3} / k_{1}\right)\left(\Delta f_{1}+\delta f_{1}\right) \\
& \vdots \\
\dot{\tilde{x}}_{n} & =\Delta f_{n}+\delta f_{n}-\left(k_{n} / k_{1}\right)\left(\Delta f_{1}+\delta f_{1}\right) .
\end{aligned}
$$

By linearizing locally with respect to the point at $\hat{\mathbf{x}}$, eq. (16) can be treated as a state observer with the following total differential form:

$$
\begin{aligned}
\dot{\tilde{x}}_{2}= & \left\{\frac{\partial f_{2}}{\partial x_{2}}-\frac{k_{2}}{k_{1}} \frac{\partial f_{1}}{\partial x_{2}}\right\} \tilde{x}_{2}+\cdots+\left\{\frac{\partial f_{2}}{\partial x_{n}}-\frac{k_{2}}{k_{1}} \frac{\partial f_{1}}{\partial x_{n}}\right\} \tilde{x}_{n} \\
& +\left(\delta f_{2}-\frac{k_{2}}{k_{1}} \delta f_{1}\right) \\
\dot{\tilde{x}}_{3}= & \left\{\frac{\partial f_{3}}{\partial x_{2}}-\frac{k_{3}}{k_{1}} \frac{\partial f_{1}}{\partial x_{2}}\right\} \tilde{x}_{2}+\cdots+\left\{\frac{\partial f_{3}}{\partial x_{n}}-\frac{k_{3}}{k_{1}} \frac{\partial f_{1}}{\partial x_{n}}\right\} \tilde{x}_{n} \\
& +\left(\delta f_{3}-\frac{k_{3}}{k_{1}} \delta f_{1}\right) \\
& \vdots \\
\dot{\tilde{x}}_{n}= & \left\{\frac{\partial f_{n}}{\partial x_{2}}-\frac{k_{n}}{k_{1}} \frac{\partial f_{1}}{\partial x_{2}}\right\} \tilde{x}_{2}+\cdots+\left\{\frac{\partial f_{n}}{\partial x_{n}}-\frac{k_{n}}{k_{1}} \frac{\partial f_{1}}{\partial x_{n}}\right\} \tilde{x}_{n} \\
& +\left(\delta f_{n}-\frac{k_{n}}{k_{1}} \delta f_{1}\right) .
\end{aligned}
$$

In the following, we first neglect the structural modeling error terms. Linearization is made on the basis of each point of the estimated states, instead of a fixed point of equilibrium, hence, the deviations considered for linearization are $\tilde{x}_{i} \mathrm{~s}$, rather than considering how far they are away from the fixed point. Consequently, this would be less restrictive compared to the extended linearization method of Baumann and Rugh (1986).

Let

$$
\dot{\tilde{\mathbf{x}}}_{r}=\left[\begin{array}{c}
\dot{\tilde{x}}_{2} \\
\dot{\tilde{x}}_{3} \\
\vdots \\
\dot{\tilde{x}}_{n}
\end{array}\right]=H(\hat{\mathbf{x}})\left[\begin{array}{c}
\tilde{x}_{2} \\
\tilde{x}_{3} \\
\vdots \\
\tilde{x}_{n}
\end{array}\right]
$$

where $\tilde{\mathbf{x}}_{r} \in \mathbf{R}^{n-1}$ and

$$
H(\hat{\mathbf{x}}) \triangleq\left[\begin{array}{ccc}
\frac{\partial f_{2}}{\partial x_{2}}-\frac{k_{2}}{k_{1}} \frac{\partial f_{1}}{\partial x_{2}} & \cdots & \frac{\partial f_{2}}{\partial x_{n}}-\frac{k_{2}}{k_{1}} \frac{\partial f_{1}}{\partial x_{n}} \\
\frac{\partial f_{3}}{\partial x_{2}}-\frac{k_{3}}{k_{1}} \frac{\partial f_{1}}{\partial x_{2}} & \cdots & \frac{\partial f_{3}}{\partial x_{n}}-\frac{k_{3}}{k_{1}} \frac{\partial f_{1}}{\partial x_{n}} \\
\vdots & \ddots & \vdots \\
\frac{\partial f_{n}}{\partial x_{2}}-\frac{k_{n}}{k_{1}} \frac{\partial f_{1}}{\partial x_{2}} & \cdots & \frac{\partial f_{n}}{\partial x_{n}}-\frac{k_{n}}{k_{1}} \frac{\partial f_{1}}{\partial x_{n}}
\end{array}\right]
$$

To keep the eigenvalues of the observer invariant, the switching gains, $\mathbf{k}_{r} \triangleq\left[\begin{array}{llll}k_{2} & k_{3} & \cdots & k_{n}\end{array}\right]^{\mathrm{T}}$, can be directly calculated by a specific formula depicted below. From eq. (19), it becomes

$$
\begin{aligned}
\boldsymbol{H}(\hat{\mathbf{x}}) & =\left[\begin{array}{ccc}
\frac{\partial f_{2}}{\partial x_{2}} & \cdots & \frac{\partial f_{2}}{\partial x_{n}} \\
\vdots & \ddots & \vdots \\
\frac{\partial f_{n}}{\partial x_{2}} & \cdots & \frac{\partial f_{n}}{\partial x_{n}}
\end{array}\right]\left[\begin{array}{c}
\left(k_{2} / k_{1}\right) \\
\vdots \\
\left(k_{n} / k_{1}\right)
\end{array}\right]\left[\begin{array}{lll}
\frac{\partial f_{1}}{\partial x_{2}} & \cdots & \frac{\partial f_{1}}{\partial x_{n}}
\end{array}\right] \\
& \triangleq \nabla_{\mathbf{x}_{r}} \mathbf{f}_{r}-\boldsymbol{\beta} \nabla_{\mathbf{x}_{r}} f_{1} \\
& \triangleq \mathbf{A}_{\mathbf{r}}-\boldsymbol{\beta} \mathbf{c}_{r} .
\end{aligned}
$$




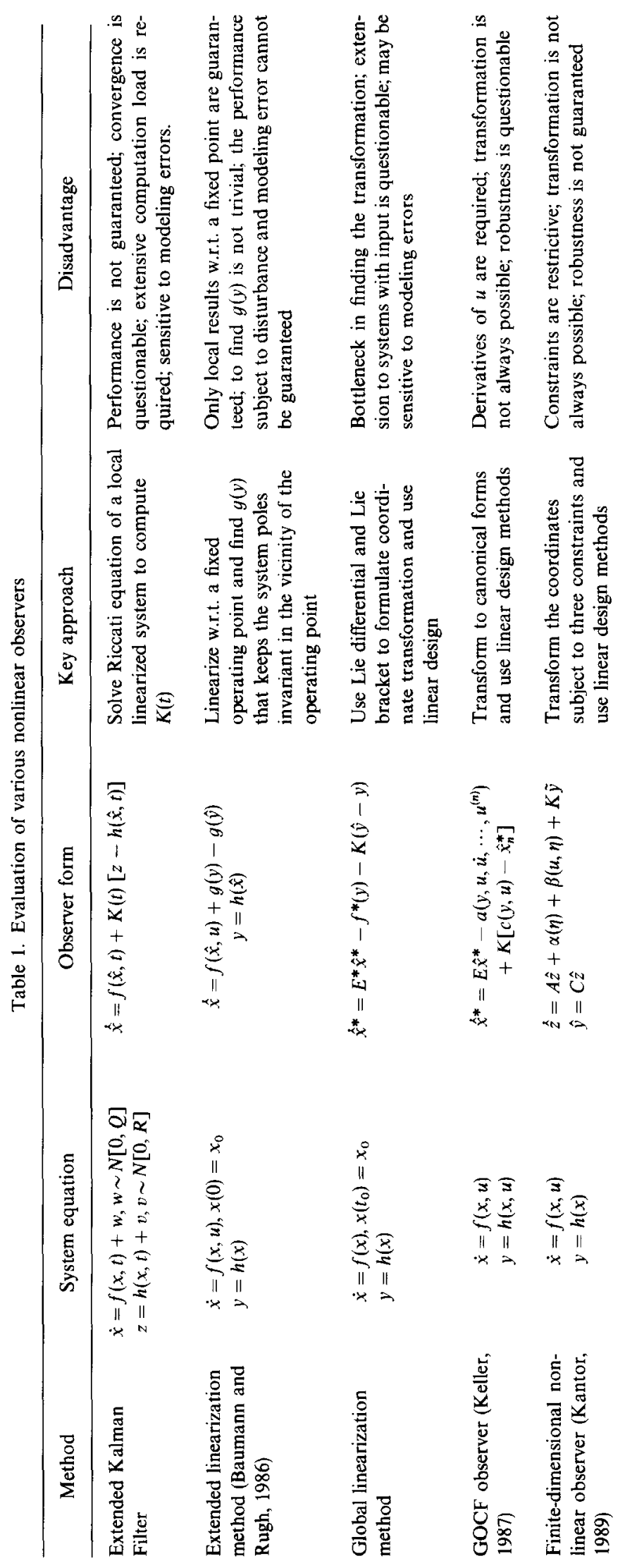



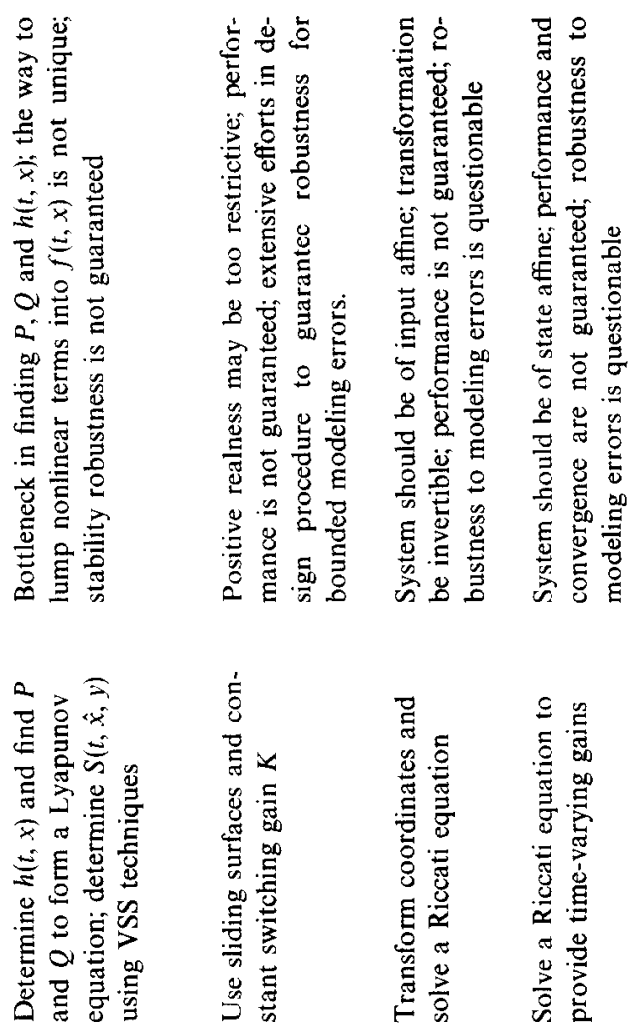

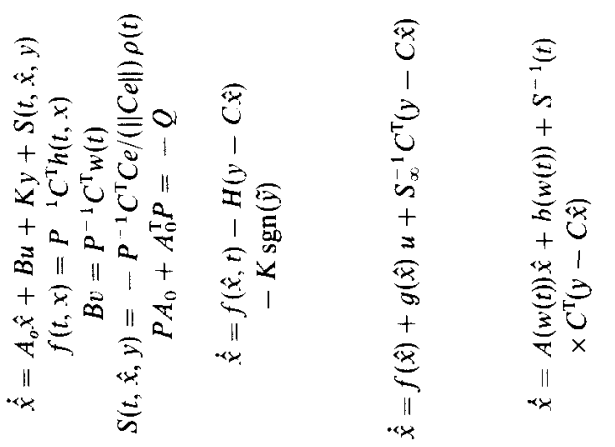
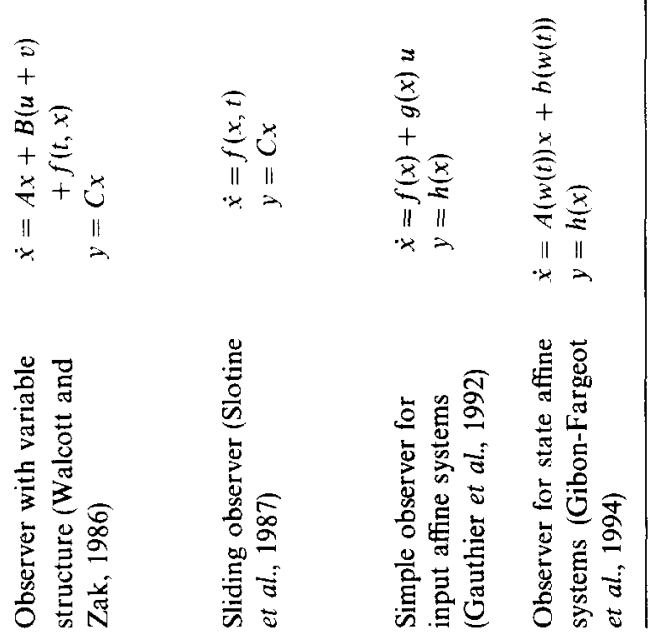
Let $\mathbf{a}_{r} \triangleq\left[\begin{array}{llll}a_{2} & a_{3} & \cdots & a_{n}\end{array}\right]$ be the coefficient vector of the characteristic equation of $\mathbf{A}_{r}$, i.e.

$\operatorname{det}\left(s \mathbf{I}-\mathbf{A}_{r}\right)=s^{n-1}+a_{2} s^{n-2}+a_{3} s^{n-3}+\cdots+a_{n}=0$.

Also, define

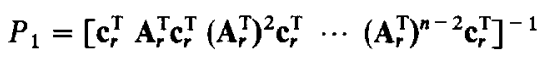

and

$$
R_{r}=\left[\begin{array}{ccccc}
1 & 0 & \cdots & 0 & 0 \\
a_{2} & 1 & \cdots & 0 & 0 \\
\vdots & \vdots & \ddots & \vdots & \vdots \\
a_{n-2} & a_{n-3} & \cdots & 1 & 0 \\
a_{n-1} & a_{n-2} & \cdots & a_{2} & 1
\end{array}\right]
$$

If $\alpha_{r} \triangleq\left[\begin{array}{llll}\alpha_{2} & \alpha_{3} & \cdots & \alpha_{n}\end{array}\right]$ is set to be the coefficient vector of the desired characteristic equation of $H(\hat{\mathbf{x}})$, then the Bass-Gura formula (Kailath, 1980) can be used to derive the following:

$$
\beta^{\mathrm{T}}=\left(\boldsymbol{\alpha}_{r}-\mathbf{a}_{r}\right)\left(R_{r}^{-1}\right)^{\mathrm{T}} P_{1} .
$$

Therefore, when the poles of the reduced-order sliding observer are assigned, the designed switching gains can be calculated as

$$
\mathbf{k}_{r}=k_{1} \boldsymbol{\beta}=k_{1} P_{1}^{T} R_{r}^{-1}\left(\boldsymbol{\alpha}_{r}-\mathbf{a}_{r}\right)^{\mathbf{T}} .
$$

Furthermore, to eliminate undesirable chattering effect, it is practical to replace the sign function in eq. (7) by a saturation function, $\operatorname{sat}(s / \phi)$, which is defined as

$$
\operatorname{sat}(s / \phi)= \begin{cases}s / \phi & \text { if }|s / \phi| \leqslant 1 \\ \operatorname{sgn}(s / \phi) & \text { if }|s / \phi|>1 .\end{cases}
$$

It is clear that by using the sliding condition for the state corresponding to the measured output, state $x_{1}$ is forced to lie on a sliding surface. As a result, the reduced-order observer has invariant dynamic poles and is guaranteed to converge asymptotically. On the other hand, it should be noted that this approach would inevitably introduce tracking error. Hence, a trade-off between tracking accuracy and control power has to be achieved by suitably choosing the boundary layer thickness $\phi$ (Slotine and Sastry, 1983).

\section{NOMINAL CONVERGENCE AND ROBUSTNESS}

Substituting $x_{1}$, corresponding to the measured output, onto a defined sliding surface, we further show that even for the remaining states the observer is stable and is robust to modeling errors.

Let the Lyapunov function $V$ be of the form

$$
V(\tilde{\mathbf{x}})=\frac{1}{2}\left\{\tilde{x}_{1}^{2}+\tilde{x}_{2}^{2}+\cdots+\tilde{x}_{n}^{2}\right\} .
$$

Then we have

$$
\dot{V}(\tilde{\mathbf{x}})=\tilde{x}_{1} \cdot \dot{\tilde{x}}_{1}+\tilde{x}_{2} \cdot \dot{\tilde{x}}_{2}+\cdots+\tilde{x}_{n} \cdot \dot{x}_{n} .
$$

Thus, if $x_{1}$ is in sliding mode, we would have $\dot{\tilde{x}}_{1}=0$ and all the first time derivatives of $\tilde{x}_{i}, i \geqslant 2$, will be given by eqs (8) and (16) so that

$$
\begin{aligned}
& \dot{V}(\tilde{\mathbf{x}})=\tilde{x}_{2} \cdot \dot{\tilde{x}}_{2}+\cdots+\tilde{x}_{n} \cdot \dot{\tilde{x}}_{n} \\
& =\tilde{x}_{2}\left[\Delta f_{2}+\delta f_{2}-k_{2} \operatorname{sgn}\left(\tilde{x}_{1}\right)\right]+\cdots \\
& +\tilde{x}_{n}\left[\Delta f_{n}+\delta f_{n}-k_{n} \operatorname{sgn}\left(\tilde{x}_{1}\right)\right] \\
& =\left\{\tilde{x}_{2}\left[\Delta f_{2}-\frac{k_{2}}{k_{1}} \Lambda f_{1}\right]+\tilde{x}_{2}\left[\delta f_{2}-\frac{k_{2}}{k_{1}} \delta f_{1}\right]\right\} \\
& +\cdots+\left\{\tilde{x}_{n}\left[\Delta f_{n}-\frac{k_{n}}{k_{1}} \Delta f_{1}\right]+\tilde{x}_{n}\left[\delta f_{n}-\frac{k_{n}}{k_{1}} \delta f_{1}\right]\right\} \\
& =\left[\begin{array}{llll}
\tilde{x}_{2} & \tilde{x}_{3} & \cdots & \tilde{x}_{n}
\end{array}\right] \\
& \times\left[\begin{array}{ccc}
\frac{\partial f_{2}}{\partial x_{2}}-\frac{k_{2}}{k_{1}} \frac{\partial f_{1}}{\partial x_{2}} & \cdots & \frac{\partial f_{2}}{\partial x_{n}}-\frac{k_{2}}{k_{1}} \frac{\partial f_{1}}{\partial x_{n}} \\
\frac{\partial f_{3}}{\partial x_{2}}-\frac{k_{3}}{k_{1}} \frac{\partial f_{1}}{\partial x_{2}} & \cdots & \frac{\partial f_{3}}{\partial x_{n}}-\frac{k_{3}}{k_{1}} \frac{\partial f_{1}}{\partial x_{n}} \\
\vdots & \ddots & \vdots \\
\frac{\partial f_{n}}{\partial x_{2}}-\frac{k_{n}}{k_{1}} \frac{\partial f_{1}}{\partial x_{2}} & \cdots & \frac{\partial f_{n}}{\partial x_{n}}-\frac{k_{n}}{k_{1}} \frac{\partial f_{1}}{\partial x_{n}}
\end{array}\right]\left[\begin{array}{c}
\tilde{x}_{2} \\
\tilde{x}_{3} \\
\vdots \\
\tilde{x}_{n}
\end{array}\right] \\
& +\left[\begin{array}{llll}
\tilde{x}_{2} & \tilde{x}_{3} & \cdots & \tilde{x}_{n}
\end{array}\right]\left[\begin{array}{c}
\delta f_{2}-\frac{k_{2}}{k_{1}} \delta f_{1} \\
\delta f_{3}-\frac{k_{3}}{k_{1}} \delta f_{1} \\
\vdots \\
\delta f_{n}-\frac{k_{n}}{k_{1}} \delta f_{1}
\end{array}\right]
\end{aligned}
$$$$
\triangleq \tilde{\mathbf{x}}_{r}^{\mathrm{T}} H(\hat{\mathbf{x}}) \tilde{\mathbf{x}}_{r}+\tilde{\mathbf{x}}_{r}^{\mathrm{T}} \boldsymbol{\delta}_{r}
$$

It has been shown that the switching gains, $k_{i}, 2 \leqslant i \leqslant n$, are calculated according to eq. (22), hence enabling matrix $H(\hat{\mathbf{x}})$ to have specific eigenvalues in the LHP. Therefore, the stability of the reduced-order observer for nominal case, i.e. $\delta \mathbf{f} \equiv \mathbf{0}$, can be guaranteed.

The issue of robustness of the observer concerns whether or not $\tilde{x}_{r}$ diverges in the presence of modeling errors. From eqs (17) and (25), it can be defined that

$$
\dot{\tilde{\mathbf{x}}}_{r}=H(\hat{\mathbf{x}}) \tilde{\mathbf{x}}_{r}+\delta_{r}
$$

Further, the solution for $\tilde{x}_{r}$ can be expressed as

$$
\tilde{\mathbf{x}}_{r}=\mathrm{e}^{H t} \tilde{\mathbf{x}}_{r}(0)+\int_{0}^{t} \mathrm{e}^{H(t-\tau)} \delta_{r} \mathrm{~d} \tau .
$$

If $\left\|\tilde{\mathbf{x}}_{r}(0)\right\| \leqslant a$ and there exist $b, \varepsilon$ and $N$ such that

$$
\|\delta \mathbf{f}\| \leqslant\left(b+N \mathrm{e}^{-\varepsilon t}\right) /\left(1+\left\|\mathbf{k}_{r}\right\| / k_{1}\right)
$$

then we have

$$
\left\|\tilde{\mathbf{x}}_{r}\right\| \leqslant a \mathrm{e}^{-\lambda_{m} t}+\frac{b}{\lambda_{m}}+\frac{N}{\lambda_{m}-\varepsilon} \mathrm{e}^{-\varepsilon t}
$$


where $-\lambda_{m}\left(\lambda_{m}>0\right)$ represents the greatest eigenvalue of $H(\hat{\mathbf{x}}),\left\|\tilde{\mathbf{x}}_{r}\right\|$ is the vector norm of $\tilde{\mathbf{x}}_{r}$ and $a, b$, $N$ and $\varepsilon$ are all positive constants. The derivation of eq. (28) is given in the Appendix. It may hence be concluded that

(i) $\left\|\tilde{\mathbf{x}}_{r}\right\|_{\text {i }}$ would remain bounded, if $\|\delta \mathbf{f}\| \leqslant$ $\left(b+N \mathrm{e}^{-\varepsilon t}\right) /\left(1+\left\|\mathbf{k}_{r}\right\| / k_{1}\right), b>0$ and $0<\varepsilon<i_{m}$.

(ii) $\left\|\tilde{\mathbf{x}}_{r}\right\| \rightarrow 0$, if $b=0,\|\delta \mathbf{f}\| \leqslant\left(N \mathrm{e}^{-\varepsilon t}\right) /\left(1+\left\|\mathbf{k}_{r}\right\| / k_{1}\right)$ and $0<\varepsilon<\lambda_{m}$.

In other words, the estimation errors of the reducedorder observer remain bounded if $\|\delta \mathbf{f}\|$ is bounded as given in eq. (27).

\section{SLIDING OBSERVERS FOR MULTI-OUTPUT SYSTEMS}

For multi-output systems, the construction of sliding observer can be derived directly from the SISO system by approximately the same way as described above. Nevertheless, the method of finding switching gain matrix $\mathbf{K}$ would require more sophisticated mathematical manipulations. For process control, however, we are more interested in formulating the problem as follows.

Instead of using a full matrix, we assign a block diagonal form to $\mathbf{K}$ in eq. (5). In other words, we can renumber the system and divide the system into several subsystems. Within each subsystem, the state estimations are corrected by a sign function based on the same output. The key issue would be the assignment of each pivot switching gain that would keep the output on its sliding surface, and the reduced-order system to have invariant dynamic modes. We will illustrate these procedures by using a system which has two outputs. First, according to the system

$$
\begin{aligned}
\dot{\mathbf{x}} & =\mathbf{f}(\mathbf{x}, \mathbf{u}) \\
\mathbf{y} & =\mathbf{C x}
\end{aligned}
$$

we can renumber the states such that

$$
\begin{aligned}
& y_{1}=x_{1} \\
& y_{1}=x_{l} .
\end{aligned}
$$

Partition the vector $\mathbf{x}$ into $\mathbf{x}^{a}$ and $\mathbf{x}^{b}$, so that

$$
\begin{aligned}
\dot{\mathbf{x}}^{a} & =\left[\begin{array}{llll}
\dot{x}_{1} & \dot{x}_{2} & \cdots & \dot{x}_{f-1}
\end{array}\right]^{\mathrm{T}} \\
\dot{\mathbf{x}}^{b} & =\left[\begin{array}{llll}
\dot{x}_{f} & \dot{x}_{f+1} & \cdots & \dot{x}_{n}
\end{array}\right]^{\mathbf{T}} .
\end{aligned}
$$

The observer is then formulated as follows:

$$
\begin{aligned}
& \dot{\hat{\mathbf{x}}}^{a}=\mathbf{f}^{a}\left(\hat{\mathbf{x}}^{a}, \hat{\mathbf{x}}^{b}, \mathbf{u}\right)+\mathbf{k}^{a} \operatorname{sgn}\left(y_{1}-\hat{x}_{1}\right) \\
& \dot{\hat{\mathbf{x}}}^{b}=\mathbf{f}^{b}\left(\hat{\mathbf{x}}^{a}, \hat{\mathbf{x}}^{b}, \mathbf{u}\right)+\mathbf{k}^{b} \operatorname{sgn}\left(y_{\ell}-\hat{x}_{\ell}\right)
\end{aligned}
$$

where

$$
\mathbf{k}^{a}=\left[\begin{array}{llll}
k_{1} & k_{2} & \cdots & k_{f-1}
\end{array}\right]^{\mathrm{T}} \text { and } \mathbf{k}^{b}=\left[\begin{array}{llll}
k_{\ell} & k_{t+1} & \cdots & k_{n}
\end{array}\right]^{\mathrm{T}} \text {. }
$$

Thus, we can assign two sliding surfaces as

$$
s_{1}=\tilde{x}_{1} \quad \text { and } \quad s_{2}=\tilde{x}_{i} .
$$

The resulting reduced-order system now becomes

$$
\begin{aligned}
{\left[\begin{array}{c}
\dot{\tilde{\mathbf{x}}}_{r}^{a} \\
\dot{\mathbf{x}}_{r}^{b}
\end{array}\right]=} & {\left[\begin{array}{cc}
\nabla_{\mathbf{x}_{r}} \mathbf{f}_{r}^{a} & \nabla_{\mathbf{x}_{r}^{b}} \mathbf{f}_{r}^{a} \\
\nabla_{\mathbf{x}_{r}{ }_{r} \mathbf{f}_{r}^{b}} & \nabla_{\mathbf{x}_{r}^{b}} \mathbf{f}_{r}^{b}
\end{array}\right]\left[\begin{array}{c}
\tilde{\mathbf{x}}_{r}^{a} \\
\tilde{\mathbf{x}}_{r}^{b}
\end{array}\right] } \\
& -\left[\begin{array}{cc}
\frac{\mathbf{k}_{r}^{a}}{k_{1}} & \mathbf{0} \\
\mathbf{0} & \frac{\mathbf{k}_{r}^{b}}{k_{r}}
\end{array}\right]\left[\begin{array}{ll}
\nabla_{\mathbf{x}_{r}^{a}} f_{1} & \nabla_{\mathbf{x}_{r}^{b}} f_{1} \\
\nabla_{\mathbf{x}_{r}^{a}} f & \nabla_{\mathbf{x}_{r}^{b}} f
\end{array}\right]\left[\begin{array}{c}
\tilde{\mathbf{x}}_{r}^{a} \\
\tilde{\mathbf{x}}_{r}^{b}
\end{array}\right]
\end{aligned}
$$

where

$\mathbf{k}_{r}^{a}=\left[\begin{array}{llll}k_{2} & k_{3} & \cdots & k_{/-1}\end{array}\right]^{\mathrm{T}}$ and $\mathbf{k}_{r}^{b}=\left[\begin{array}{llll}k_{\ell+1} & k_{f+2} & \cdots & k_{n}\end{array}\right]^{\mathrm{T}}$.

Therefore, we have a reduced observer pair as

$\mathbf{A}_{r}=\left[\begin{array}{ll}\nabla_{\mathbf{x}_{r}^{a}} \mathbf{f}_{r}^{a} & \nabla_{\mathbf{x}_{r}^{b}} \mathbf{f}_{r}^{a} \\ \nabla_{\mathbf{x}_{r}^{a}} \mathbf{f}_{r}^{b} & \nabla_{\mathbf{x}_{r}^{b}} \mathbf{f}_{r}^{b}\end{array}\right]$ and $\mathbf{C}_{r}=\left[\begin{array}{ll}\nabla_{\mathbf{x}_{r}^{a}} f_{1} & \nabla_{\mathbf{x}_{r}^{b}} f_{1} \\ \nabla_{\mathbf{x}_{r}^{a}} f_{f} & \nabla_{\mathbf{x}_{r}^{b}} f_{r}\end{array}\right]$

and $\mathbf{K}_{r}$ as

$$
\mathbf{K}_{r}=\left[\begin{array}{cc}
\mathbf{k}_{r}^{a} & \mathbf{0} \\
k_{1} & \mathbf{k}_{r}^{b} \\
\mathbf{0} & \frac{k_{f}}{k^{\prime}}
\end{array}\right] .
$$

We can further derive $\mathbf{k}_{r}^{a}$ and $\mathbf{k}_{r}^{b}$ from the following equality:

$$
\operatorname{det}\left\{s \mathbf{I}-\mathbf{A}_{r}+\mathbf{K}_{r} \mathbf{C}_{r}\right\}=\prod_{i=1}^{n-2}\left(s-\hat{\lambda}_{i}\right)
$$

where $\lambda_{i}, i=1,2, \cdots, n-2$, are the specified eigenvalues. Solving eq. (34) for deriving $\mathbf{k}_{r}^{a}$ and $\mathbf{k}_{r}^{b}$ would need tedious algebraic manipulations if $n$ is higher than three. Chen (1984) has provided three effective methods to find $\mathbf{K}_{r}$.

Although there may be different ways of partitioning the observer into the subsystems as given in eq. (30), however, there is little change in the reducedorder system given in eq. (31) except for the changes in configuration of $\mathbf{K}_{r}$ matrix. Hence, different decompositions would result in the same set of columns for the observability matrix of $\left[\mathbf{A}_{r}, \mathbf{C}_{\boldsymbol{r}}\right]$ pair. The changes in the system due to different partitioning of subsystems are in fact changes in the eigenvectors of $\left(\mathbf{A}_{r}-\mathbf{K}_{r} \mathbf{C}_{r}\right)$ which are associated with each dynamic mode (Chen, 1984; Lewis, 1992)

While formulating the observer for chemical processes, wherein state variables can obviously be classified into non-interacting groups, we can take advantage of this method of partitioning. Thus, eq. (30) becomes

$$
\begin{aligned}
& \dot{\hat{\mathbf{x}}}^{a}=\mathbf{f}^{a}\left(\hat{\mathbf{x}}^{a}, \mathbf{u}\right)+\mathbf{k}^{a} \operatorname{sgn}\left(y_{1}-\hat{x}_{1}\right) \\
& \dot{\hat{\mathbf{x}}}^{b}=\mathbf{f}^{b}\left(\hat{\mathbf{x}}^{a}, \hat{\mathbf{x}}^{b}, \mathbf{u}\right)+\mathbf{k}^{b} \operatorname{sgn}\left(y_{f}-\hat{x}_{f}\right) .
\end{aligned}
$$

Hence, we shall have

$$
\begin{aligned}
\operatorname{det}\{s \mathbf{I} & \left.-\mathbf{A}_{r}+\mathbf{K}_{r} \mathbf{C}_{r}\right\}=\operatorname{det}\left\{s \mathbf{I}-\nabla_{\mathbf{x}_{r}^{a}} \mathbf{f}_{r}^{a}\right. \\
& \left.+\frac{\mathbf{k}_{r}^{a}}{k_{1}} \nabla_{\mathbf{x}_{r}^{a}} f_{1}\right\} \operatorname{det}\left\{s \mathbf{I}-\nabla_{\mathbf{x}_{r}^{b}} \mathbf{f}_{r}^{b}+\frac{\mathbf{k}_{r}^{b}}{k_{f}} \nabla_{\mathbf{x}_{r}^{b}} f_{r}\right\} \\
= & \prod_{i=1}^{n-2}\left(s-\lambda_{i}\right) .
\end{aligned}
$$




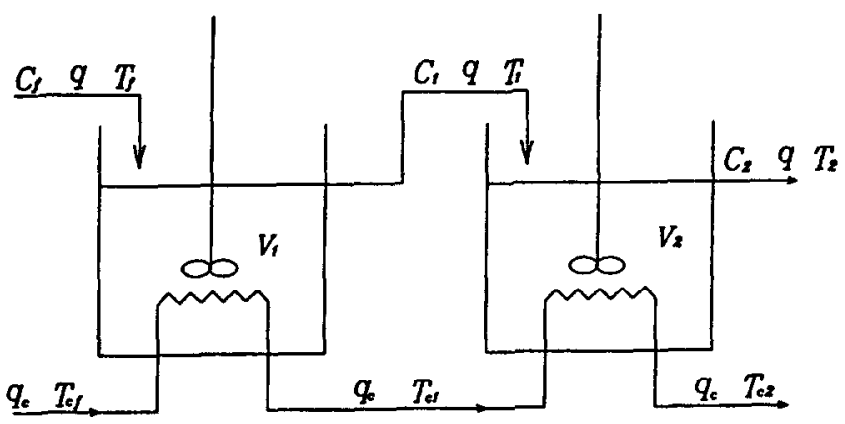

Fig. 1. The serial CSTR system.

In other words, the computations for $\mathbf{k}_{r}^{a}$ and $\mathbf{k}_{r}^{h}$ can now be decoupled. Each of the $\mathbf{k}_{r}^{a}$ and $\mathbf{k}_{r}^{b}$ can be calculated individually by using eq. (22) for each of the subsystems. In the following, we will illustrate this proposed sliding observer with an example of estimation of a chemical reaction system.

Example (State estimation for a serial chemical reaction system): Figure 1 shows the serial continuousstirred tank reactor (CSTR) system, belonging to the multi-output case and studied by Henson and Seborg (1990). The dynamic behavior of this system can be governed by the following equations:

$$
\begin{aligned}
\dot{C}_{1}= & \frac{q}{V_{1}}\left(C_{f}-C_{1}\right)-k_{0} C_{1} \exp \left(-\frac{E}{R T_{1}}\right) \\
\dot{T}_{1}= & \frac{q}{V_{1}}\left(T_{f}-T_{1}\right)+\frac{(-\Delta H) k_{0} C_{1}}{\rho c_{p}} \exp \left(-\frac{E}{R T_{1}}\right) \\
& +\frac{\rho_{c} c_{p c}}{\rho c_{p} V_{1}} q_{c}\left[1-\exp \left(-\frac{U A_{1}}{q_{c} \rho_{c} c_{p c}}\right)\right]\left(T_{c f}-T_{1}\right) \\
\dot{C}_{2}= & \frac{q}{V_{2}}\left(C_{1}-C_{2}\right)-k_{0} C_{2} \exp \left(-\frac{E}{R T_{2}}\right) \\
\dot{T}_{2}= & \frac{q}{V_{2}}\left(T_{1}-T_{2}\right)+\frac{(-\Delta H) k_{0} C_{2}}{\rho c_{p}} \exp \left(-\frac{E}{R T_{2}}\right) \\
& +\frac{\rho_{c} c_{p c}}{\rho c_{p} V_{2}} q_{c}\left[1-\exp \left(-\frac{U A_{2}}{q_{c} \rho_{c} c_{p c}}\right)\right] \\
& \times\left[T_{1}-T_{2}+\exp \left(-\frac{U A_{1}}{q_{c} \rho_{c} c_{p c}}\right)\left(T_{c f}-T_{1}\right)\right] .
\end{aligned}
$$

The nominal values of the parameters of this serial CSTR system are given in Table 2 . The state variables $x$, system output $y$ and manipulated input $u$ are defined as follows:

$$
x \triangleq\left[\begin{array}{llll}
C_{1} & T_{1} & C_{2} & T_{2}
\end{array}\right]^{\mathrm{T}}, \quad y \triangleq\left[\begin{array}{ll}
T_{1} & T_{2}
\end{array}\right]^{\mathrm{T}}, \quad u \triangleq q_{c} .
$$

Ience, the dynamic model of the serial CSTR in dimensionless form gives

$$
\begin{aligned}
& \dot{x}_{1}=1-x_{1}-k_{0} x_{1} \exp \left(-\frac{E}{R x_{2}}\right) \\
& \dot{x}_{2}=T_{f}-x_{2}+\frac{k_{0}(-\Delta H)}{\rho c_{p}} x_{1} \exp \left(-\frac{E}{R x_{2}}\right)
\end{aligned}
$$

$$
\begin{aligned}
& +0.01 u\left[1-\exp \left(-\frac{U A_{1}}{\rho_{c} c_{p c} u}\right)\right]\left(T_{c f}-x_{2}\right) \\
\dot{x}_{3}= & x_{1}-x_{3}-k_{0} x_{3} \exp \left(-\frac{E}{R x_{4}}\right) \\
\dot{x}_{4}= & x_{2}-x_{4}+\frac{k_{0}(-\Delta H)}{\rho c_{p}} x_{3} \exp \left(-\frac{E}{R x_{4}}\right) \\
& +0.01 u\left[1-\exp \left(-\frac{U A_{2}}{\rho_{c} c_{p c} u}\right)\right]\left[x_{2}-x_{4}\right. \\
& \left.+\exp \left(-\frac{U A_{1}}{\rho_{c} c_{p c} u}\right)\left(T_{c f}-x_{2}\right)\right] .
\end{aligned}
$$

It is apparent that this reacting system has two measurable outputs, i.e. it can be split into two subsystcms. Therefore, to estimate the concentrations in the reactor, one can design the sliding observer as follows:

$$
\begin{aligned}
\dot{\hat{x}}_{1}=1 & -\hat{x}_{1}-k_{0} \hat{x}_{1} \exp \left(-\frac{E}{R \hat{x}_{2}}\right)+k_{1} \operatorname{sgn}\left(\tilde{x}_{2} / \phi\right) \\
\dot{\hat{x}}_{2}= & T_{f}-\hat{x}_{2}+\frac{k_{0}(-\Delta H)}{\rho c_{p}} \hat{x}_{1} \exp \left(-\frac{E}{R \hat{x}_{2}}\right) \\
& +0.01 u\left[1-\exp \left(-\frac{U A_{1}}{\rho_{c} c_{p c} u}\right)\right]\left(T_{c f}-\hat{x}_{2}\right) \\
& +k_{2} \operatorname{sgn}\left(\tilde{x}_{2} / \phi\right) \\
\dot{\hat{x}}_{3}= & \hat{x}_{1}-\hat{x}_{3}-k_{0} \hat{x}_{3} \exp \left(-\frac{E}{R \hat{x}_{4}}\right)+k_{3} \operatorname{sgn}\left(\tilde{x}_{4} / \phi\right) \\
\dot{\hat{x}}_{4}= & \hat{x}_{2}-\hat{x}_{4}+\frac{k_{0}(-\Delta H)}{\rho c_{p}} \hat{x}_{3} \exp \left(-\frac{E}{R \hat{x}_{4}}\right) \\
& +0.01 u\left[1-\exp \left(-\frac{U A_{2}}{\rho_{c} c_{p c} u}\right)\right]\left[\hat{x}_{2}-\hat{x}_{4}\right. \\
& \left.+\exp \left(-\frac{U A_{1}}{\rho_{c} c_{p c} u}\right)\left(T_{c f}-\hat{x}_{2}\right)\right]+k_{4} \operatorname{sgn}\left(\tilde{x}_{4} / \phi\right)
\end{aligned}
$$

where $\tilde{x}_{4} \triangleq x_{4}-\hat{x}_{4}$. The switching gains $k_{2}$ and $k_{4}$ are determined according to eq. (12). The other two switching gains can be directly calculated according to eq. (22). 
Table 2. Nominal parameter values for the serial CSTR system

\begin{tabular}{cc}
\hline Variable & Nominal value \\
\hline$q$ & $100 \mathrm{l} / \mathrm{min}$ \\
$V_{1}=V_{2}$ & $100 \mathrm{l}$ \\
$C_{f}$ & $1 \mathrm{~mol} / 1$ \\
$T_{f}$ & $350 \mathrm{~K}$ \\
$T_{c f}$ & $350 \mathrm{~K}$ \\
$k_{0}$ & $7.2 \times 10^{10} \mathrm{~min}^{-1}$ \\
$E / R$ & $10,000 \mathrm{~K}$ \\
$(-\Delta H)$ & $4.78 \times 10^{4} \mathrm{~J} / \mathrm{mol}^{\prime}$ \\
$p=\rho_{c}$ & $1000 \mathrm{~g} / 1$ \\
$c_{p}=c_{p c}$ & $0.239 \mathrm{~J} / \mathrm{g} \mathrm{K}$ \\
$U A_{1}=U A_{2}$ & $1.67 \times 10^{5} \mathrm{~J} / \mathrm{min} \mathrm{K}$ \\
\hline
\end{tabular}

For this simulation case, the process input is given as

$$
u=\left\{\begin{aligned}
90 \mathrm{l} / \mathrm{min} & \text { if } 0 \leqslant t<5 \mathrm{~min} \\
100 \mathrm{l} / \mathrm{min} & \text { if } t \geqslant 5 \mathrm{~min} .
\end{aligned}\right.
$$

Initial conditions for the true states and the estimated states are set as $\left[\begin{array}{llllll}0.085 & 442 & 0.005 & 450\end{array}\right]^{\mathrm{T}}$ and $\left[\begin{array}{llll}0.05 & 442 & 0 & 450\end{array}\right]^{\mathrm{T}}$, respectively, and the switching gains of the measurable outputs are set as $k_{2}=80$ and $k_{4}=25$. A suitable boundary layer thickness $\phi=0.01$ is also selected. It is apparent that the values of species concentrations should either be positive or zero. Hence, when the estimated concentration values, i.e. $\hat{x}_{1}$ and $\hat{x}_{3}$, are lower than zero, they are set to be zeros. Estimation results, which are depicted in Figs 2 and 3, demonstrate the performance of the proposed sliding observer for state estimations.

\section{APPLICATION TO PROCESS CONTROL}

The sliding observers developed in the previous section offer advantages such as: ease of construction and on-line implementation, robustness to modeling errors and guaranteed convergence. However, prior to applying such an observer to process control, two issues nced to be resolved. One is the observability of states in the closed-loop and other is the stability after combination.

For the former, consider a nonlinear system as given in eq. (1), its local observability at $\mathbf{x} \in \mathbf{X}$ depends on whether the following condition is satisfied (Keller, 1987):

$$
\operatorname{rank}\left[\begin{array}{c}
\left(\frac{\partial}{\partial \mathbf{x}} M^{0} \mathbf{h}(\mathbf{x}, \mathbf{u})\right)^{\mathrm{T}} \\
\left(\frac{\partial}{\partial \mathbf{x}} M^{1} \mathbf{h}(\mathbf{x}, \mathbf{u})\right)^{\mathrm{T}} \\
\left(\frac{\partial}{\partial \mathbf{x}} M^{2} \mathbf{h}(\mathbf{x}, \mathbf{u})\right)^{\mathrm{T}} \\
\vdots \\
\left(\frac{\partial}{\partial \mathbf{x}} \boldsymbol{M}^{n-1} \mathbf{h}(\mathbf{x}, \mathbf{u})\right)^{\mathrm{T}}
\end{array}\right]=n
$$

where $\mathbf{X}$ is an open subset of $\mathbf{R}^{n}$. The operator $M$ is defined as

$$
\begin{aligned}
M^{\circ} h_{i}(\mathbf{x}, \mathbf{u})= & h_{i}(\mathbf{x}) \\
M^{1} h_{i}(\mathbf{x}, \mathbf{u})= & {\left[\frac{\partial}{\partial \mathbf{x}} h_{i}(\mathbf{x})\right]^{\mathrm{T}}[\mathbf{f}(\mathbf{x}, \mathbf{u})] } \\
& \vdots \\
M^{k} h_{i}(\mathbf{x}, \mathbf{u})= & {\left[\frac{\partial}{\partial \mathbf{x}} M^{k-1} h_{i}(\mathbf{x}, \mathbf{u})\right]^{\mathrm{T}} \mathbf{f}(\mathbf{x}, \mathbf{u}) } \\
& +\left[\frac{\partial}{\partial \mathbf{u}} M^{k-1} h_{i}(\mathbf{x}, \mathbf{u})\right]^{\mathrm{T}} \mathbf{\mathbf { u }}
\end{aligned}
$$

where $i=1,2, \ldots, p$ and $k=1,2, \ldots, n$.

It can be clearly seen that the rank of this observability matrix would be a function of $\mathbf{x}$ and $\mathbf{u}$. For open loop use, the values of $\mathbf{u}$ can be freely assigned. Nevertheless, in closed-loop control, it is important to note that the control inputs are no longer free variables. However, since $\mathbf{u}$ cannot be explicitly separated from $\mathbf{x}$, it becomes difficult to determine whether it is feasible to construct an observer for closed-loop control under certain feedback law.

A simplified expression for local observability condition at each local point of $(\mathbf{x}, \mathbf{u})$ can be given for non-affine systems as follows:

$$
\operatorname{Rank}\left[\begin{array}{c}
\lambda I-\frac{\partial}{\partial \mathbf{x}} \mathbf{f}(\mathbf{x}, \mathbf{u}) \\
\frac{\partial}{\partial \mathbf{x}} \mathbf{h}(\mathbf{x})
\end{array}\right]=n \quad \forall \lambda .
$$

The above condition is a direct result of the assumption that at $(\mathbf{x}, \mathbf{u})$ the system is locally observable and hence the system can be locally linearized by using the PBH rank test. To construct an observer for closedloop control, it is however necessary to examine the observability condition of eq. (41) over a domain of $\mathbf{x}$ as well as that of feasible control input.

Some special cases having simpler observability condition are as follows:

Input affine systems: A large number of dynamic processes have the system equations in form of

$$
\begin{aligned}
& \dot{\mathbf{x}}=\mathbf{f}(\mathbf{x})+\mathbf{g}(\mathbf{x}) \mathbf{u} \\
& \mathbf{y}=\mathbf{h}(\mathbf{x}) .
\end{aligned}
$$

For such systems, a sufficient condition for local observability at some $\mathbf{x}_{0} \in \mathbf{X}$ would be to find $n$ independent row vectors among the following (Vidyasagar, 1993):

$$
\begin{gathered}
{\left[\frac{\partial}{\partial \mathbf{x}} L_{\mathbf{v}_{s}} \cdots L_{\mathbf{v}_{1}} h_{i}\left(\mathbf{x}_{0}\right), \quad s \geqslant 0,1 \leqslant i \leqslant p,\right.} \\
\left.\mathbf{v}_{1}, \cdots, \mathbf{v}_{s} \in\left\{\mathbf{f}, \mathbf{g}_{1}, \cdots, \mathbf{g}_{m}\right\}\right]
\end{gathered}
$$



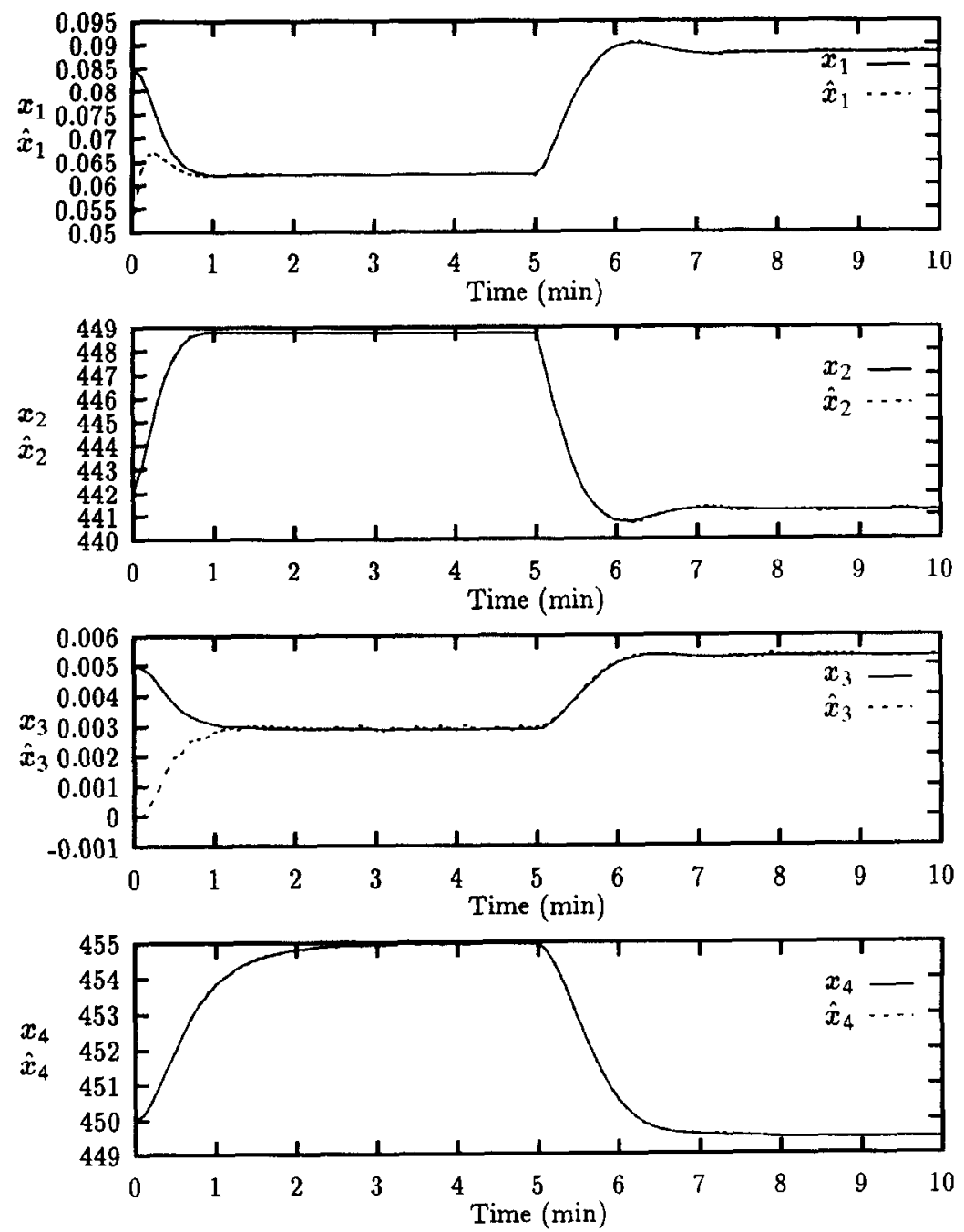

Fig. 2. Estimation results of the serial CSTR system under sliding observer with observer poles $p_{O 1}=p_{02}=-5$.

As the vector $\mathbf{u}$ does not explicitly appear in the above formulation, to construct an observer for closed-loop system, it would be requircd to examine the observability condition over a given domain of $\mathbf{x}$ only.

State affine systems: For some chemical reaction systems (Gibon-Fargeot et al., 1994), the dynamic equations can be described by the following equations:

$$
\begin{aligned}
\dot{\mathbf{x}} & =A(u(t), y(t)) \mathbf{x}+\mathbf{b}(u(t), y(t)) \\
y & =\mathbf{c x} .
\end{aligned}
$$

The observability, for this kind of systems, would depend on whether or not the $(A, \mathrm{c})$ pair is observable.

The other criterion to be considered while using the observer for nonlinear process control is whether incorporation of the proposed sliding observer into a control system would causc only stability problem To answer this question, we shall consider a general observer of eq. (2). The closed-loop system will then be governed by the following equations:

$$
\begin{aligned}
\dot{\mathbf{x}} & =\mathbf{f}(\mathbf{x}, u) \\
\dot{\hat{\mathbf{x}}} & =\mathbf{f}(\hat{\mathbf{x}}, u)+\Phi(y-\hat{y}) \\
y & =\mathbf{c x}
\end{aligned}
$$

where $\Phi$ designates a correcting function which is used in the observer. If we define the difference between the true states and the estimated states as

$$
\tilde{\mathbf{x}}=\mathbf{x}-\hat{\mathbf{x}}
$$

then the above governing equations become

$$
\begin{aligned}
\dot{\mathbf{x}} & =\mathbf{f}(\mathbf{x}, u) \\
\dot{\tilde{\mathbf{x}}} & =\mathbf{f}(\mathbf{x}, u)-\mathbf{f}(\hat{\mathbf{x}}, u)-\Phi(\mathbf{c} \tilde{\mathbf{x}})
\end{aligned}
$$

In linear systems, it is easy to show that $\tilde{\mathbf{x}}$ and $\mathbf{x}$ have independent dynamic modes, and the dynamic modes of the overall system would be a combination of both sets. Thus, incorporating a stable observer into a stable state feedback control system would result in 

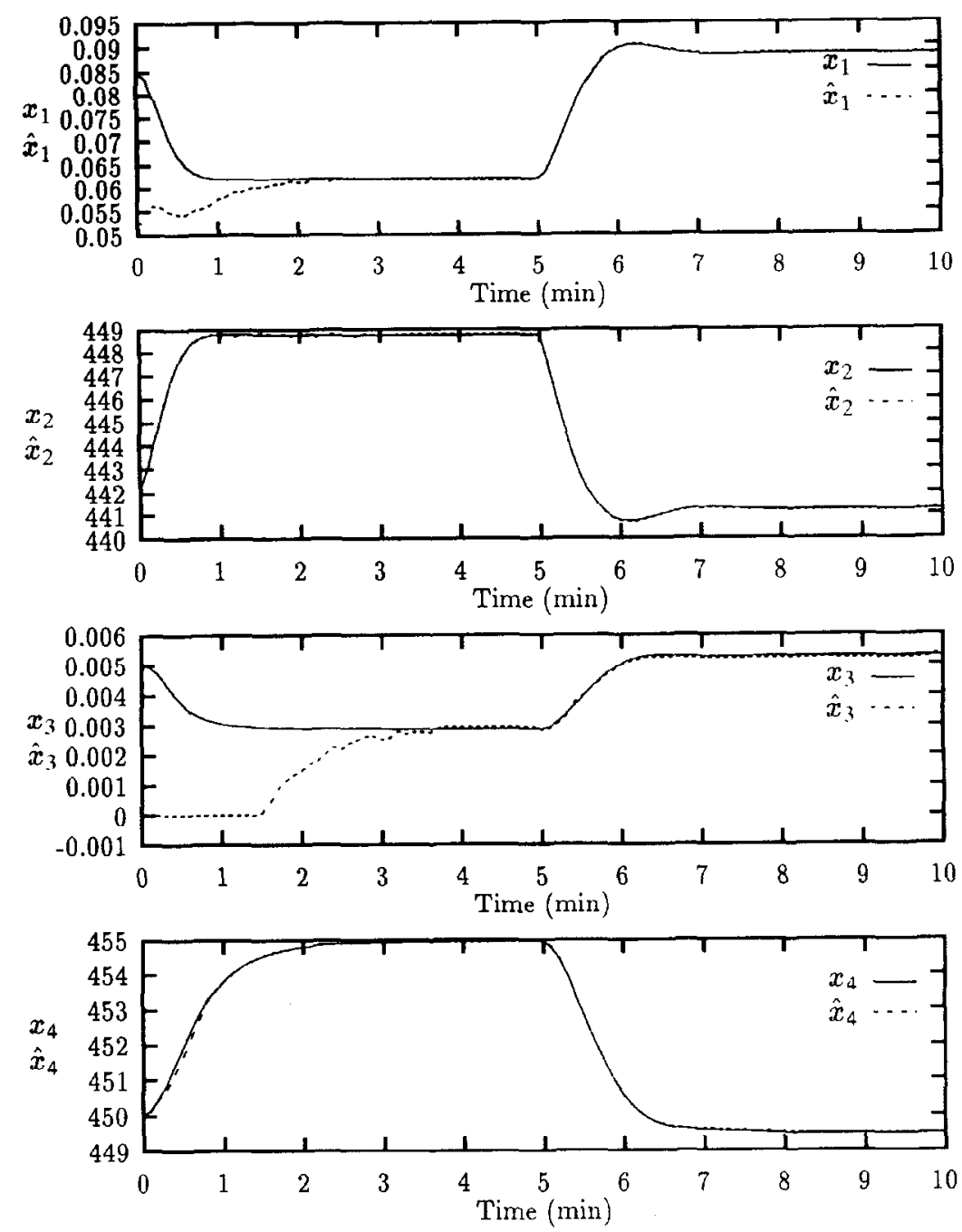

Fig. 3. Estimation results of the serial CSTR system under sliding observer with observer poles $p_{O 1}=p_{O 2}=-2$.

the formulation of a stable closed-loop system. In general, it is difficult to reach such a conclusion for nonlinear systems. However, it is essential to know that, if the estimated states converge asymptotically to their true values with bounded transient errors, then whether a stable state feedback system remains stable after incorporating the proposed sliding observer.

According to the result given in the previous section, eq. (48) for the system that uses the proposed sliding observer now becomes

$$
\begin{aligned}
\dot{\mathbf{x}} & =\mathbf{f}(\mathbf{x}, \boldsymbol{u}(\mathbf{x}-\tilde{\mathbf{x}})) \\
\dot{\tilde{\mathbf{x}}}_{r} & =\left(\nabla_{\mathbf{x}_{r}} \mathbf{f}_{r}-\boldsymbol{\beta} \nabla_{\mathbf{x}_{r}} f_{1}\right) \tilde{\mathbf{x}}_{r} .
\end{aligned}
$$

It can be obviously seen from eq. (50) that $\tilde{\mathbf{x}}_{r}$ is independent of $\mathbf{x}$. On the other hand, eq. (49) can be linearized with respect to $\tilde{\mathbf{x}}$ at the point of $\tilde{\mathbf{x}}=0$, i.e. $\hat{\mathbf{x}}=\mathbf{x}$, as

$\dot{\mathbf{x}}=\mathbf{f}(\mathbf{x}, u(\mathbf{x}-\tilde{\mathbf{x}}))=\mathbf{f}(\mathbf{x}, u(\mathbf{x}))+\left.\frac{\partial \mathbf{f}}{\partial u} \frac{\partial u}{\partial \tilde{\mathbf{x}}}\right|_{\tilde{\mathbf{x}}=0} \tilde{\mathbf{x}}+o\left(\tilde{\mathbf{x}}^{2}\right)$.
Moreover, within the neighborhood of some equilibrium point $\mathbf{x}_{0}$, we can further derive that

$$
\begin{aligned}
\dot{\mathbf{x}}= & \mathbf{f}\left(\mathbf{x}_{0}, u\left(\mathbf{x}_{0}\right)\right)+\left.\left(\nabla_{\mathbf{x}} \mathbf{f}+\frac{\partial \mathbf{f}}{\partial u} \frac{\partial u}{\partial \mathbf{x}}\right)\right|_{\mathbf{x}=\mathbf{x}_{0}}\left(\mathbf{x}-\mathbf{x}_{0}\right) \\
& +W\left(\mathbf{x}_{0}, \tilde{\mathbf{x}}\right)\left(\mathbf{x}-\mathbf{x}_{0}\right)+o\left(\tilde{\mathbf{x}}^{2}\right) \\
\approx & \left(\left.\nabla_{\mathbf{x}} \mathbf{f}\right|_{\mathbf{x}-\mathbf{x}_{0}}+\left.\frac{\partial \mathbf{f}}{\partial u} \frac{\partial u}{\partial \mathbf{x}}\right|_{\mathbf{x}=\mathbf{x}_{0}}+W\left(\mathbf{x}_{0}, \tilde{\mathbf{x}}\right)\right)\left(\mathbf{x}-\mathbf{x}_{0}\right)
\end{aligned}
$$

where

$W\left(\mathbf{x}_{0}, \tilde{\mathbf{x}}\right) \triangleq \nabla_{\mathbf{x}}(Q(\mathbf{x}) \tilde{\mathbf{x}})=\left.\nabla_{\mathbf{x}} \mathbf{q}_{1}\right|_{\mathbf{x}=\mathbf{x}_{0}} \tilde{\mathbf{x}}_{1}+\left.\nabla_{\mathbf{x}} \mathbf{q}_{2}\right|_{\mathbf{x}=\mathbf{x}_{0}} \tilde{\mathbf{x}}_{2}$

$$
+\cdots+\left.\nabla_{\mathbf{x}} \mathbf{q}_{n}\right|_{\mathbf{x}=\mathbf{x}_{0}} \tilde{x}_{n}
$$

and

$$
\left.Q(\mathbf{x}) \triangleq \frac{\partial \mathbf{f}}{\partial u} \frac{\partial u}{\partial \tilde{\mathbf{x}}}\right|_{\tilde{\mathbf{x}}=0}=\left[\begin{array}{llll}
\mathbf{q}_{1}(\mathbf{x}) \mathbf{q}_{2}(\mathbf{x}) & \cdots & \mathbf{q}_{n}(\mathbf{x})
\end{array}\right] .
$$


Let

$$
\begin{aligned}
\tilde{A}\left(\mathbf{x}_{0}, \tilde{\mathbf{x}}\right) & \left.\triangleq \nabla_{\mathbf{x}} \mathbf{f}\right|_{\mathbf{x}=\mathbf{x}_{0}}+\left.\frac{\partial \mathbf{f}}{\partial u} \frac{\partial u}{\partial \mathbf{x}}\right|_{\mathbf{x}=\mathbf{x}_{0}}+W\left(\mathbf{x}_{0}, \tilde{\mathbf{x}}\right) \\
& \triangleq A\left(\mathbf{x}_{0}\right)+W\left(\mathbf{x}_{0}, \tilde{\mathbf{x}}\right) .
\end{aligned}
$$

where

$$
\tilde{A}\left(\mathbf{x}_{0}, \mathbf{0}\right)=A\left(\mathbf{x}_{0}\right)
$$

The local stability of this system can be guaranteed by studying the eigenvalues of the following linearized matrix at the equilibrium point $\mathbf{x}_{0}$ :

$$
\tilde{A}\left(\mathbf{x}_{0}, \tilde{\mathbf{x}}\right)=A\left(\mathbf{x}_{0}\right)+W\left(\mathbf{x}_{0}, \tilde{\mathbf{x}}\right) .
$$

It is known that, without loss of generality, one can set $\mathbf{x}_{0}$ as $\mathbf{0}$. Thus, we have

$$
\tilde{A}(\mathbf{0}, \tilde{\mathbf{x}})=A(\mathbf{0})+W(\mathbf{0}, \tilde{\mathbf{x}}) .
$$

Notice that the ideal system, whose all states are accessible, is stable such that all the eigenvalues of $\boldsymbol{A}(\mathbf{0})$ lie in the LHP. The effect of the observer on its stability is limited to the extra term, $W(\mathbf{0}, \tilde{\mathbf{x}})$. The stability of this observer-based closed-loop system should be discussed in light of the following theorem.

Theorem (Perturbation theorem for the eigenvalue, Stewart and Sun, 1990): Let $\lambda$ be a simple eigenvalue of the matrix $A$, with right and left eigenvectors $v$ and $\omega$, and let $\overline{\mathrm{A}}=\mathrm{A}+\mathrm{E}$ be a perturbation of $\mathrm{A}$. Then there exists a unique eigenvalue $\tilde{\lambda}$ of $\tilde{\mathrm{A}}$ such that

$$
\tilde{\lambda}=\lambda+\frac{\omega^{H} E v}{\omega^{H} v}+o\left(\|E\|^{2}\right)
$$

Based on the above theorem, we have

$$
\tilde{\lambda}[\tilde{A}]=\lambda[A]-\frac{\omega^{H} W v}{\omega^{H} v}+o\left(\|W\|^{2}\right) .
$$

We can therefore conclude that if

$$
\max _{i}\left|\frac{\omega^{H} W v}{\omega^{H} v}\right|<\left|\lambda_{i}\right| \quad \forall i
$$

the incorporation of this sliding observer would not affect its nominal stability which is derived from the ideal system. As can be seen from eq. (51), as $\tilde{\mathbf{x}}$ approaches zero as time passes, the observer based system will convert to an ideal system.

Although the stability of such an observer-based system cannot be said to be totally independent of the observer, the stability condition can be achieved more easily since this sliding observer provides the guaranteed convergence as in the previous analysis. As a result, if the control using full state feedback is globally stable within a defined domain of $\mathbf{x}$, it should be possible to incorporate a carefully designed observer which will not cause a stability problem. Consequently, design of the state feedback control and the observer can be implemented separately. In other words, one can design a stable control system by assuming that all state variables are fed back. A sliding observer as depicted above can then be constructed. Following this guideline, we illustrate in the following subsections the use of such a sliding observer for controlling chemical processes.

\subsection{Setpoint tracking of chemical reactor control}

It is known that all control systems are nonlinear to a certain extent. Hence, the development and application of nonlinear control algorithms are attracting great attention in the recent years. Here, we utilize the GLC structure to deal with the servo control problem of an isothermal CSTR. The GLC approach was first proposed by Kravaris and Chung (1987) for obtaining the linear relationship between the transformed inputs and process outputs for nonlinear systems (Bequette, 1991). Therefore, the linear control theory, which is well-developed for the linear systems, can be utilized to complete the controller design.

For a minimum phase nonlinear system with relative order $r$, the state feedback control law

$$
u=\frac{v-L_{f}^{r} h(x)}{L_{g} L_{f}^{r-1} h(x)}
$$

can directly transform the original nonlinear system into

$$
y^{(r)}=v
$$

Furthermore, the new manipulated input $v$ is set to be

$$
\begin{aligned}
v= & -\theta_{r} y^{(r-1)}-\theta_{r-1} y^{(r-2)} \\
& -\cdots-\theta_{2} y-\theta_{1}\left(y-y_{s p}\right) .
\end{aligned}
$$

The closed-loop transfer function (CLTF) of the system can then be obtained by combining eqs (58) and (59) as

$$
\frac{y(s)}{y_{s p}(s)}-\frac{\theta_{1}}{s^{r}+\theta_{r} s^{r-1}+\cdots+\theta_{2} s+\theta_{1}} .
$$

It may be mentioned here that most of the nonlinear control techniques utilize state feedback compensator laws. Hence, the employment of state observers becomes necessary. In the following, we solve the estimation and control problems of the chemical reaction system by applying the sliding observer developed above.

Let us consider a well-mixed CSTR with isothermal reaction as

$$
\mathrm{A} \rightleftharpoons \mathrm{B} \rightarrow \mathrm{C} .
$$

By denoting the concentrations of species A, B and $\mathrm{C}$ as $x_{1}, x_{2}$ and $x_{3}$, respectively, material balances for this CSTR are described by the following dimensionless equations:

$$
\begin{aligned}
\dot{x}_{1} & =1-x_{1}-D_{1} x_{1}+D_{2} x_{2}^{2} \\
\dot{x}_{2} & =D_{1} x_{1}-x_{2}-D_{2} x_{2}^{2}-D_{3} x_{2}^{2}+u \\
\dot{x}_{3} & =D_{3} x_{2}^{2}-x_{3} \\
y & =h(x)=x_{3} .
\end{aligned}
$$


Since the system has relative order $r=2$, according to eqs $(58)$ and (59), we have

$$
\ddot{y}=v=-\theta_{2} \dot{y}-\theta_{1}\left(y-y_{s p}\right) .
$$

Then the corresponding nonlinear control law derived from eq. (57) gives in form of

$$
\begin{aligned}
& \dot{\hat{x}}_{1}=1-\hat{x}_{1}-D_{1} \hat{x}_{1}+D_{2} \hat{x}_{2}^{2}+k_{1} \operatorname{sat}\left(\tilde{x}_{3} / \phi\right) \\
& \dot{\hat{x}}_{2}=D_{1} \hat{x}_{1}-\hat{x}_{2}-D_{2} \hat{x}_{2}^{2}-D_{3} \hat{x}_{2}^{2}+u+k_{2} \operatorname{sat}\left(\tilde{x}_{3} / \phi\right)
\end{aligned}
$$

$\dot{\hat{x}}_{3}=D_{3} \hat{x}_{2}^{2}-\hat{x}_{3}+k_{3} \operatorname{sat}\left(\tilde{x}_{3} / \phi\right)$

$u=\frac{-2 D_{3} \hat{x}_{2}\left(D_{1} \hat{x}_{1}-\hat{x}_{2}-D_{2} \hat{x}_{2}^{2}\right)+\left(D_{3} \hat{x}_{2}^{2}-y\right)-\theta_{2}\left(D_{3} \hat{x}_{2}^{2}-y\right)-O_{1}\left(y-y_{s p}\right)}{2 D_{3} \hat{x}_{2}}$

where $\theta_{1}$ and $\theta_{2}$ are tuning parameters of the GLC approach. The overall closed-loop transfer function is thus obtained from eq. (60) as

$$
\frac{y(s)}{y_{s p}(s)}=\frac{\theta_{1}}{s^{2}+\theta_{2} s+\theta_{1}} .
$$

Furthermore, the estimated values of the unmeasurable states, $\hat{x}_{1}$ and $\hat{x}_{2}$, in the nonlinear control law, i.e. eq. (63), are provided by the proposed sliding observer where $\tilde{x}_{3}=x_{3}-\hat{x}_{3}$ and the switching gains are determined from eqs (12) and (22).

During the simulations, we choose the process parameters as $D_{1}=3, D_{2}=0.5$ and $D_{3}=1$ and set the initial conditions of the true states $x(0)$ and the estimated states $\hat{x}(0)$ as $\left[\begin{array}{llll}0.356 & 0.921 & 0.848\end{array}\right]^{\mathrm{T}}$ and $\left[\begin{array}{lll}0.5 & 1.0 & 0.848\end{array}\right]^{\mathrm{T}}$, respectively. 'Two tuning parameters of the GLC approach are placed at $\theta_{1}=4$ and $\theta_{2}=4$. In this study, the control objective is to make the
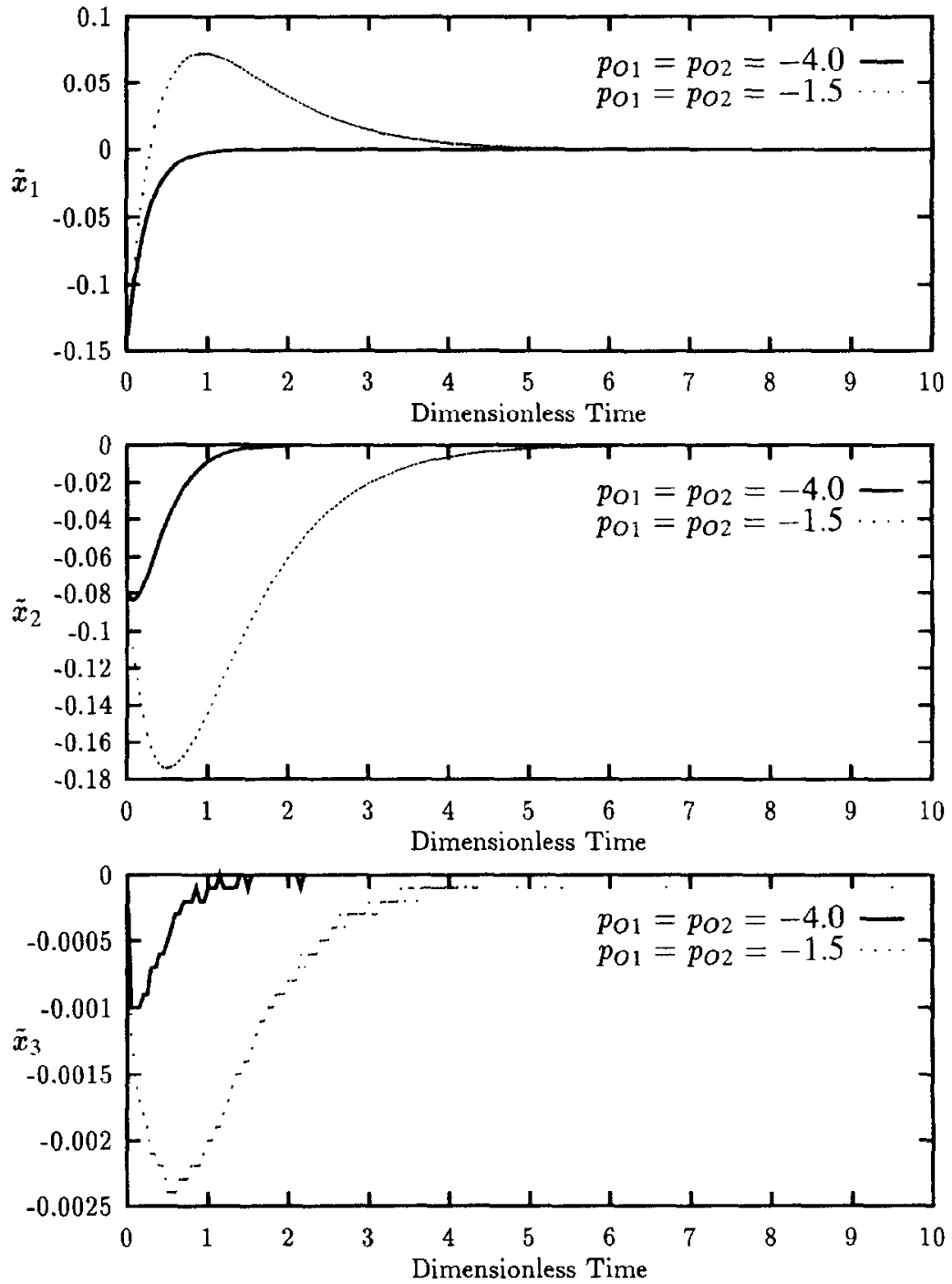

Fig. 4. Results of estimation errors for the isothermal CSTR system under sliding observer with observer poles $p_{O 1}=p_{O 2}=-4$ and -1.5 . 
dimensionless concentration $x_{3}$ track its setpoint $y_{s p}=0.75$ as soon as possible. Let the switching gain $k_{3}=1.5$ and the boundary layer thickness $\phi=0.01$. From the results of estimation errors shown in Fig. 4, it is clear that the unmeasurable concentrations can be effectively estimated by the proposed sliding observer. The servo control responses shown in Fig. 5 demonstrate the tracking performance of this sliding observer. Furthermore, it may be noted that the assigned poles of the reduced-order sliding observer do influence the convergence rate for state estimation.

Moreover, when the modeling errors occur due to parametric uncertainty, the proposed sliding observer can still perform well by setting this parameter as an unknown state variable.

6.2. Disturbance rejection for chemical reactor control

The presence of unknown disturbance in the process would prevent $\tilde{x}_{i}$ from converging to zero. As a result, the estimated states deviate from their true values which, in turn, would degrade the control performance. One way to reject such unknown disturbance is to include it in the observer as an extra state variable. For constant disturbance, this extra state serves as an integrator, i.e. for SISO systems, we have

$$
\dot{\mathbf{x}}=\mathbf{f}\left(\mathbf{x}, x_{n+1}=d, u\right), \quad \dot{x}_{n+1}=0, \quad y=x_{1}
$$

where $d$ is an unknown disturbance. A sliding observer can be constructed to estimate the unmeasured states, $x_{2}, \cdots, x_{n+1}$, including the unknown disturbance.

The matrix that corresponds to $H(\hat{\mathbf{x}})$ in eq. (19) would now become

$$
\begin{aligned}
& H^{*}\left(\hat{\mathbf{x}}_{\boldsymbol{r}}, \hat{x}_{n+1}\right)= \\
& \quad\left[\begin{array}{cc}
\boldsymbol{\nabla}_{\mathbf{x}_{r}} \mathbf{f}_{\mathbf{r}}-\boldsymbol{\beta}\left[\nabla_{\mathbf{x}_{\mathbf{r}}} f_{1}\right] & \nabla_{\mathbf{x}_{n+1}} \mathbf{f}-\boldsymbol{\beta}\left[\nabla_{\mathbf{x}_{n+1}} f_{1}\right] \\
-\psi\left[\nabla_{\mathbf{x}_{r}} f_{1}\right] & -\psi\left[\nabla_{\mathbf{x}_{n+1}} f_{1}\right]
\end{array}\right] .
\end{aligned}
$$

The existence of parameter sets of $\beta$ and $\psi$ of such a system would depend on the observability pair $\left(A^{*}, c^{*}\right)$ of the following nature:

$$
\begin{aligned}
A^{*} & =\left[\begin{array}{cc}
\nabla_{\mathbf{x}_{r}} \mathbf{f}_{r} & \nabla_{\mathbf{x}_{n+1}} \mathbf{f}_{r} \\
\mathbf{0}_{1 \times(n-1)} & 0_{1 \times 1}
\end{array}\right], \\
c^{*} & =\left[\begin{array}{ll}
\nabla_{\mathbf{x}_{r}} f_{1} & \nabla_{x_{n+1}} f_{1}
\end{array}\right] .
\end{aligned}
$$

If the pair $\left(\nabla_{\mathbf{x}_{r}} \int_{1}, \nabla_{\mathrm{x}_{\mathrm{r}}} \mathbf{f}_{r}\right)$ is observable, a necessary and sufficient condition for this augmented system to be observable is

$$
\operatorname{rank}\left[\begin{array}{cc}
\nabla_{\mathbf{x}_{r}}^{\mathbf{T}} \mathbf{f}_{r} & \boldsymbol{\nabla}_{\mathbf{x}_{r}}^{\mathbf{T}} f_{1} \\
\mathbf{\nabla}_{\mathbf{x}_{n+1}}^{\mathbf{T}} \mathbf{f}_{\boldsymbol{r}} & \mathbf{V}_{\mathbf{x}_{n+1}}^{\mathbf{T}} f_{1}
\end{array}\right]-n .
$$

Equation (69) is a result of applying the observability condition (Morari and Stephanopoulos, 1980) to the $\left(A^{*}, c^{*}\right)$ pair. For multi-output systems, the
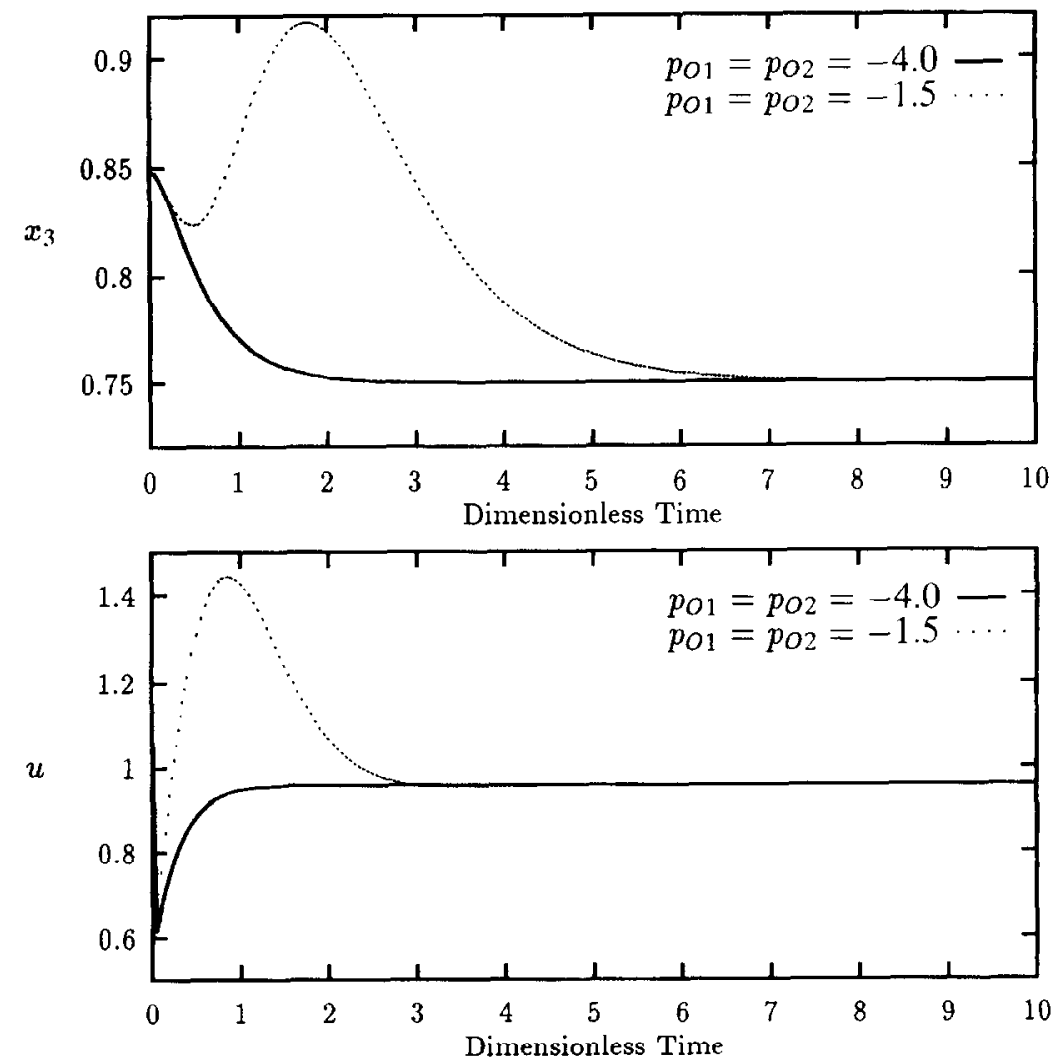

Fig. 5. Servo control responses of the isothermal CSTR system under sliding observer with observer poles $p_{01}=p_{02}=-4$ and -1.5 . 
number of unknown disturbances to be included in an observer is at the most equal to the number of system outputs.

In the following example, we consider a well-mixed CSTR with first-order, irreversible, exothermic reaction. Material and energy balances for such exothermic CSTR are described by

$$
\begin{aligned}
\frac{\mathrm{d} C}{\mathrm{~d} t}= & \frac{q}{V}\left(C_{f}-C\right)-k_{0} C \exp \left(-\frac{E}{R T}\right) \\
\frac{\mathrm{d} T}{\mathrm{~d} t}= & \frac{q}{V}\left(T_{f}-T\right)+\frac{(-\Delta H)}{\rho c_{p}} k_{0} C \exp \left(-\frac{E}{R T}\right) \\
& +\frac{U A_{H}}{\rho c_{p} V}\left(T_{c}-T\right) .
\end{aligned}
$$

where $C$ and $T$ represent the reactant exit concentration and reactor temperature, respectively. Let the four im- portant dimensionless parameters be denoted as

$$
\begin{array}{ll}
D_{4}=\frac{E}{R T_{f}}, & D_{5}=\frac{U A_{H}}{\rho c_{p} q} \\
D_{6}=\frac{k_{0} V \mathrm{e}^{-D_{4}}}{q}, & D_{7}=\frac{(-\Delta H) C_{f 0} D_{4}}{\rho c_{p} T_{f}} .
\end{array}
$$

The corresponding dimensionless variables are defined as

$$
\begin{gathered}
x_{1}=\frac{C}{C_{f 0}}, \quad x_{2}=\frac{T-T_{f}}{T_{f}} D_{4}, \quad d_{1}=\frac{C_{f}}{C_{f 0}}, \tau=\frac{q}{V} t \\
u=\frac{T_{c}-T_{f}}{T_{f}} D_{4} D_{5}, \quad y=C, \quad y_{m}=T
\end{gathered}
$$

where $d_{1}$ represents the unknown disturbance and $C_{f o}$ is the nominal value of the feed composition. After introducing the dimensionless quantities given
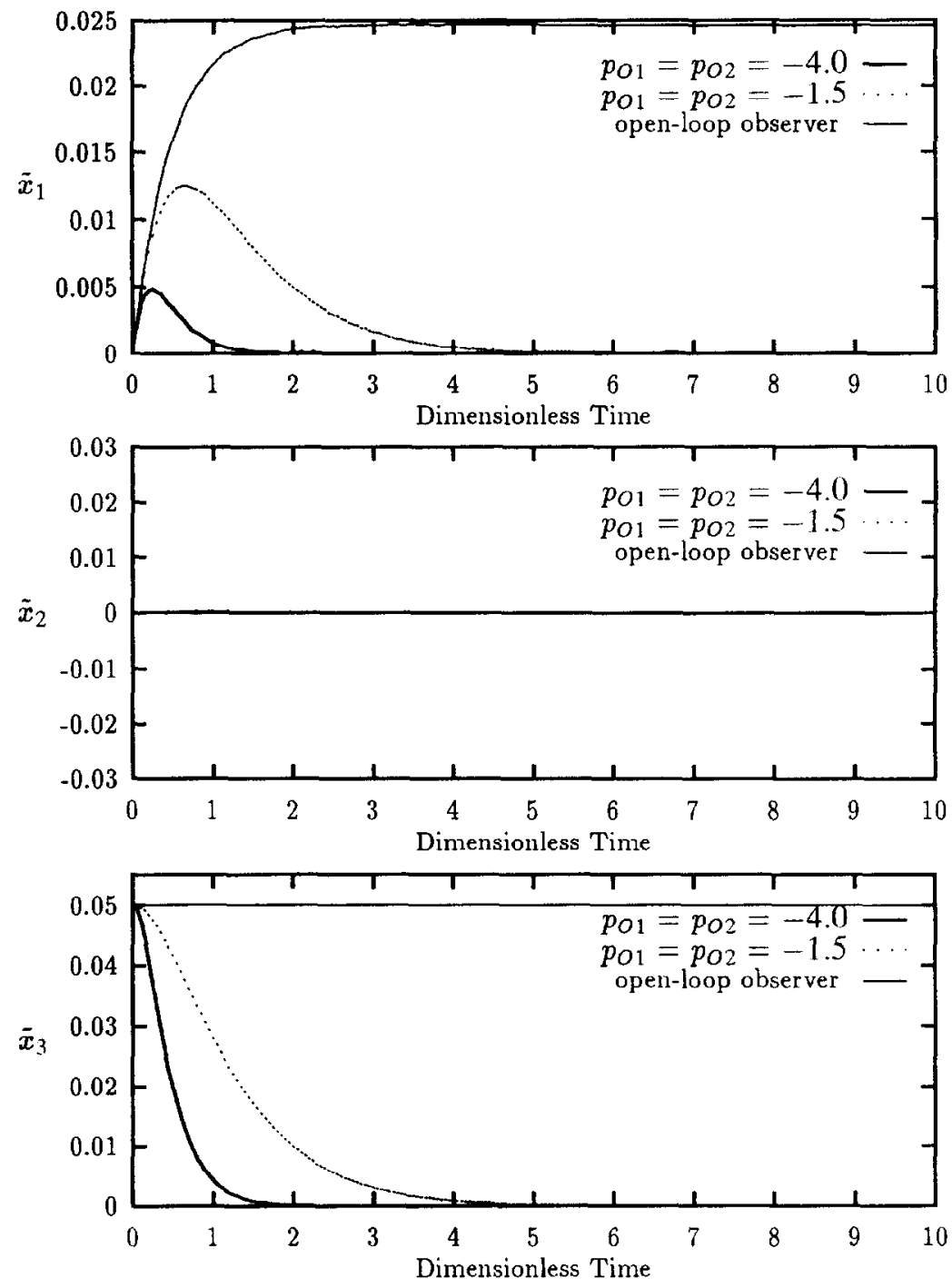

Fig. 6. Results of estimation errors for the exothermic CSTR system under sliding observer with observer poles $p_{O 1}=p_{O 2}=-4$ and -1.5 and under the open-loop observer. 

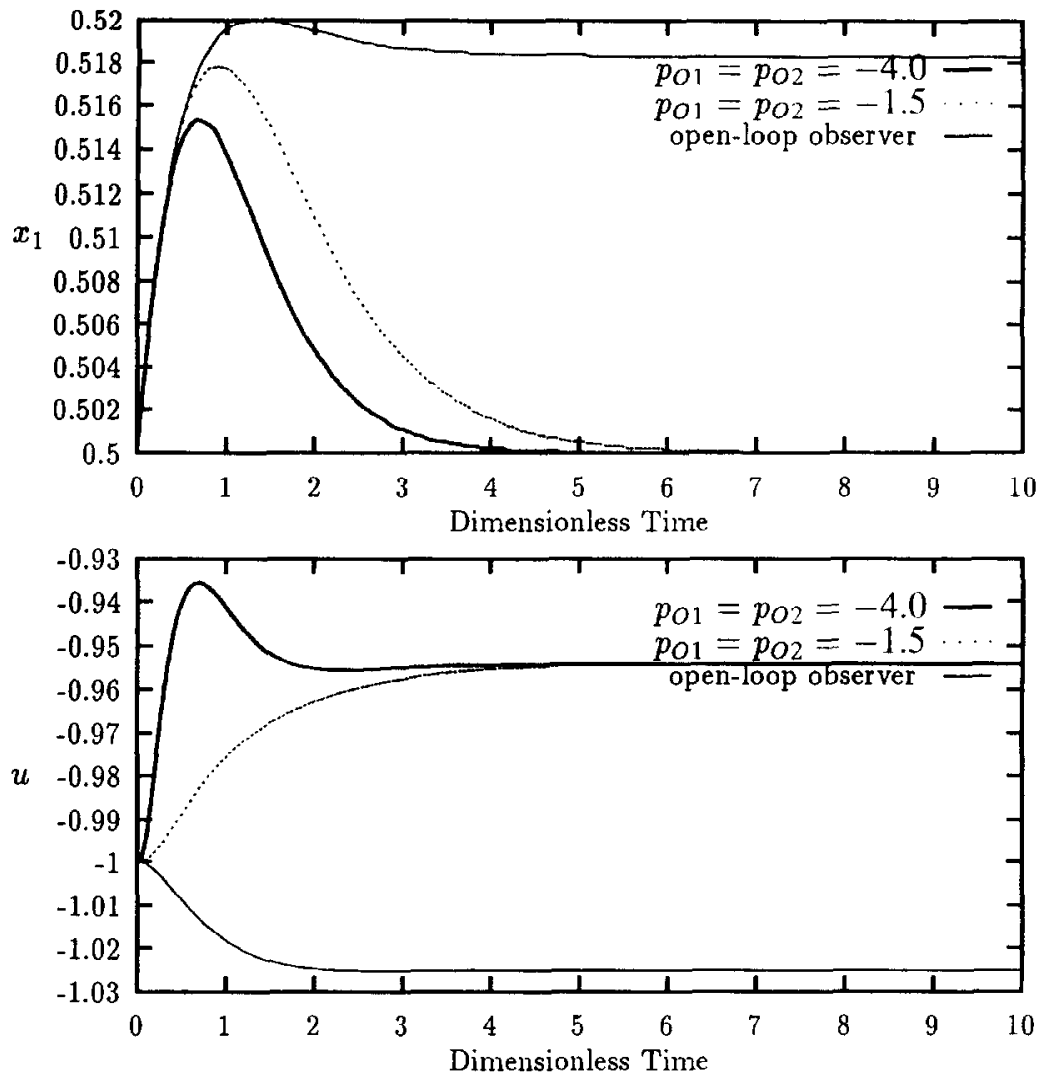

Fig. 7. Results of disturbance rejection of the exothermic CSTR system under sliding observer with observer poles $p_{O 1}=p_{O 2}=-4$ and -1.5 and under the open-loop observer.

above, the resulting normalized model is governed as

$$
\begin{aligned}
& \dot{x}_{1}=d_{1}-x_{1}-D_{6} x_{1} \exp \left(\frac{x_{2}}{1+x_{2} / D_{4}}\right) \\
& \dot{x}_{2}=D_{6} D_{7} x_{1} \exp \left(\frac{x_{2}}{1+x_{2} / D_{4}}\right)-\left(1+D_{5}\right) x_{2}+u
\end{aligned}
$$

$$
y=x_{1}, \quad y_{m}-x_{2} .
$$

In this case, the unknown disturbance $d_{1}$ is regarded as another state variable. According to eq. (73), we have

$$
\dot{x}_{1}=x_{3}-x_{1}-D_{6} x_{1} \exp \left(\frac{x_{2}}{1+x_{2} / D_{4}}\right) \triangleq f_{1}(x)
$$

where $y_{m}$ is the system measurement and

$$
\begin{aligned}
& f_{1}(x)=x_{3}-x_{1}-D_{6} x_{1} \exp \left(\frac{x_{2}}{1+x_{2} / D_{4}}\right) \\
& f_{2}(x)=D_{6} D_{7} x_{1} \exp \left(\frac{x_{2}}{1+x_{2} / D_{4}}\right)-\left(1+D_{5}\right) x_{2} \\
& g_{2}(x)=1 .
\end{aligned}
$$

We may now apply the GLC structure to solve the concentration control problem of the exothermic CSTR. The relative order $r$ of this CSTR is equal to 2; thus, let

$$
\ddot{y}=v=-\theta_{2} \dot{y}-\theta_{1}\left(y-y_{s p}\right) .
$$

Then the corresponding nonlinear control law can be directly derived from eqs (74) and (76):

$u=\frac{\left\{\theta_{2}-1-D_{6} \exp \left[y_{m} /\left(1+y_{m} / D_{4}\right)\right]\right\} f_{1}(\hat{x})-\left\{D_{6} \hat{x}_{1}\left(1+y_{m} / D_{4}\right)^{-2} \exp \left[y_{m} /\left(1+y_{m} / D_{4}\right)\right]\right\} f_{2}(\hat{x})+\theta_{1}\left(\hat{x}_{1}-y_{s p}\right)}{D_{6} \hat{x}_{1}\left(1+y_{m} / D_{4}\right)^{-2} \exp \left[y_{m} /\left(1+y_{m} / D_{4}\right)\right]}$

$$
\dot{x}_{2}=D_{6} D_{7} x_{1} \exp \left(\frac{x_{2}}{1+x_{2} / D_{4}}\right)-\left(1+D_{5}\right) x_{2}+u
$$

where $\theta_{1}$ and $\theta_{2}$ are tuning parameters of the GLC approach. The overall closed-loop transfer function can be obtained directly from eq. (76) as

$$
\triangleq f_{2}(x)+g_{2}(x) u
$$

$\dot{x}_{3}=0, \quad y=x_{1}, \quad y_{m}=x_{2}$.

$$
\frac{y(s)}{y_{s p}(s)}=\frac{\theta_{1}}{s^{2}+\theta_{2} s+\theta_{1}} .
$$


Furthermore, the estimated values of the unmeasurable states, $\hat{x}_{1}$ and $\hat{x}_{3}$, in the nonlinear control law, given by eq. (77), are provided by the proposed sliding observer in form of

$\dot{\hat{x}}_{1}=\hat{x}_{3}-\hat{x}_{1}-D_{6} \hat{x}_{1} \exp \left(\frac{\hat{x}_{2}}{1+\hat{x}_{2} / D_{4}}\right)+k_{1} \operatorname{sat}\left(\tilde{x}_{2} / \phi\right)$

$\dot{\hat{x}}_{2}=D_{6} D_{7} \hat{x}_{1} \exp \left(\frac{\hat{x}_{2}}{1+\hat{x}_{2} / D_{4}}\right)-\left(1+D_{5}\right) \hat{x}_{2}$

$$
+u+k_{2} \operatorname{sat}\left(\tilde{x}_{2} / \phi\right)
$$

$\dot{\hat{x}}_{3}=+k_{3} \operatorname{sat}\left(\tilde{x}_{2} / \phi\right)$.

where $\tilde{x}_{2}=x_{2}-\hat{x}_{2}$ and the switching gains are determined from eqs (12) and (22).

For comparisons, an open-loop observer is used to estimate the unmeasurable states by directly integrating the following differential equations:

$$
\begin{aligned}
& \dot{\hat{x}}_{1}=\hat{x}_{3}-\hat{x}_{1}-D_{6} \hat{x}_{1} \exp \left(\frac{y_{m}}{1+y_{m} / D_{4}}\right) \\
& \dot{\hat{x}}_{3}=0 .
\end{aligned}
$$

For the exothermic CSTR, the four process parameters in eq. (71) are chosen as $D_{4}=5, D_{5}=0.5$, $D_{6}=1$ and $D_{7}=2$. Initial conditions for the true statcs $x(0)$ and the estimated states $\hat{x}(0)$ arc set as $\left[\begin{array}{lll}0.5 & 0 & 1.05\end{array}\right]^{\mathrm{T}}$ and $\left[\begin{array}{lll}0.5 & 0 & 1.0\end{array}\right]^{\mathrm{T}}$, respectively. It should be noted that, in this example, the control objective is to make the dimensionless concentration $x_{1}$ remain on its setpoint $y_{s p}=0.5$ in the face of the unknown disturbance. By applying the GLC approach, the two tuning parameters are set as $\theta_{1}=4$ and $\theta_{2}=4$. Further, the switching gain $k_{2}$ and the boundary layer thickness $\phi$ are set to be 1.5 and 0.01 , respectively. Figure 6 reveals the existence of offset for estimation of the states for load change since the open-loop observer cannot provide correct estimated states. On the other hand, the response results shown in Fig. 7 demonstrate good robustness features of the reducedorder sliding observer in face of existence of an unknown disturbance.

\section{CONCLUDING REMARKS}

A sliding observer, which behaves like a reducedorder observer, is presented. This observer has been shown to overcome some major difficulties involved in constructing nonlinear observers for state estimations, especially for nonlinear process control. To achieve this, the switching gains in the observer are made to be time-varying. Convergence of the estimation is analyzed by using Lyapunov stability theorems. Robustness conditions, which would guarantee the observer to have a bounded error norm when facing modeling error, are also derived. The advantages of this proposed sliding observer include: simple and less restrictive design and construction, no need of extensive computations during its implementation, no requirement of canonical transformation, achievement of desired performance by allocating the observer poles, knowledge of convergence and robustness of the estimation to the designer, etc. Potential uses of this sliding observer towards servo-tracking and disturbance rejection for process control are discussed. Estimation of unmeasurable states and control of chemical reactor are illustrated.

\section{Acknowledgement}

Financial support from the National Science Council of the Republic of China(NSC-84-2214-E002-036) is gratefully acknowledged.

\section{NOTATION}

$a, b \quad$ positive constants

$A_{1}, A_{2}, A_{H}$ heat transfer area

A $\quad$ matrix in eq. (20) or eq. (32)

$\tilde{A}\left(\mathbf{x}_{\boldsymbol{o}}, \tilde{\mathbf{x}}\right) \quad$ matrix in eq. (53)

$c_{p} \quad$ heat capacity

$c_{r} \quad$ vector in eq. (20)

$C$ concentration

$C_{r} \quad$ matrix in eq. (32)

d disturbance

$D_{1} \sim D_{7} \quad$ process parameters of the reaction system

E activation energy

f, $\mathbf{g}$ vectors of nonlinear functions

$F \quad$ positive constant

$\Delta f \quad$ defined as $\mathbf{f}(\mathbf{x}, u)-\mathbf{f}(\hat{\mathbf{x}}, u)$

h output functions

$H(\hat{\mathbf{x}}) \quad$ matrix in eq. (19)

$\Delta H \quad$ heat of reaction

$J \quad$ Jacobian matrix

$k_{0} \quad$ specific reaction rate constant

$K \quad$ time-varying gain matrix

$L \quad$ Lie operator

$M \quad$ operator defined in eq. (42)

$N \quad$ positive constant

$p_{O 1}, p_{O 2}$ poles of the reduced-order sliding observer

$P_{1} \quad$ inverse of the transpose of the observability matrix of $\left[\mathbf{A}_{r}, \mathbf{c}_{r}\right]$ pair

$q \quad$ feed flow rate

$Q(\mathbf{x}) \quad$ matrix in eq. (52)

$r \quad$ relative order

$R \quad$ ideal gas constant

$\mathbf{R} \quad$ real scalar field

$\mathbf{R}^{n} \quad$ n-dimensional real vector field

$R_{r} \quad$ lower triangular Toeplitz matrix with first column $\left[\begin{array}{lllll}1 & a_{2} & \cdots & a_{n-2} & a_{n-1}\end{array}\right]$

sliding surface

saturation function

sign function

time

temperature

input vector

overall heat transfer coefficient

transformed control variable

reactor volume

Lyapunov function candidate 


$\begin{array}{ll}W\left(\mathbf{x}_{o}, \tilde{\mathbf{x}}\right) & \text { defined as } \nabla_{\mathbf{x}}(Q(\mathbf{x}) \tilde{\mathbf{x}}) \\ \mathbf{x} & \text { state vector } \\ \mathbf{y} & \text { output vector } \\ y_{m} & \text { measured output } \\ \mathbf{z} & \text { state vector }\end{array}$

Greek letters

$\begin{array}{ll}\boldsymbol{\alpha} & \triangleq\left[\alpha_{2} \alpha_{3} \cdots \alpha_{n}\right] \\ \boldsymbol{\beta} & \text { vector defined in eq. (20) } \\ \gamma & \text { coefficient in eq. (15) } \\ \delta f & \text { modeling error due to structural devi } \\ & \text { ation } \\ \delta_{r} & \text { vector defined in eq. (25) } \\ \varepsilon & \text { positive constant } \\ \eta & \text { positive constant } \\ \theta & \text { tuning parameters of the GLC approach } \\ \lambda & \text { eigenvalues } \\ \boldsymbol{v} & \text { vector } \\ \rho & \text { density } \\ \sigma & \text { vector defined in eq. (5) } \\ \Phi & \text { correcting function in eqs (2) and (47) } \\ \phi & \text { boundary layer thickness } \\ \psi & \text { positive constant } \\ \omega & \text { vector }\end{array}$

\section{Subscripts}

$r \quad$ reduced-order system

\section{Superscripts \\ estimated value deviation value}

\section{REFERENCES}

Alvarez-Ramirez, J. (1995) Observers for a class of continuous tank reactors via temperature measurement. Chem. Engng Sci. 50, 1393-1399.

Baumann, W. T. and Rugh, W. J. (1986) Feedback control of nonlinear systems by extended linearization. IEEE Trans. Automat. Control AC-31, $40-46$.

Bequette, B. W. (1991) Nonlinear control of chemical processes: a review. Ind. Engng Chem. Res. 30, 1391-1413.

Bestle, D. and Zeitz, M. (1983) Canonical form observer design for non-linear time-variable systems. Int. J. Control 38, 419-431.

Canudas de Wit, C. and Slotine, J.-J. E. (1991) Sliding observer for robot manipulators. Automatica 27, 859-864.

Chen, C. T. (1984) Linear System Theory and Design. Holt, Rinehart and Winston, New York, U.S.A.

Ding, X., Frank, P. M. and Guo, L. (1990) Nonlinear observer design via an extended observer canonical form. Systems Control Lett. 15, 313-322.

Gibon-Fargeot, A. M., Hammouri, H. and Celle, F. (1994) Nonlinear observers for chemical reactors. Chem. Engng Sci. 49, 2287-2300

Henson, M. A. and Seborg, D. E. (1990) Input-output linearization of general nonlinear processes. A.I.Ch.E. J. 36, 1753-1757.

Hunt, L. R., Su, R. and Meyer, G. (1983) Global transformations of nonlinear systems. IEEE Trans. Automat. Control AC-28, 24-31.
Kailath, T. (1980) Linear Systems. Prentice-Hall, Englewood Cliffs, NJ, U.S.A.

Kantor, J. C. (1989) A finite dimensional nonlinear observer for an exothermic stirred-tank reactor. Chem. Engng Sci. 44, 1503-1510.

Keller, H. (1987) Non-linear observer design by transformation into a generalized observer canonical form. Int. J. Control 46, 1915-1930.

Kravaris, C. and Chung, C. B. (1987) Nonlinear state feedback synthesis by global input/output linearization. A.I.Ch.E. J. 33, 592-603.

Lee, P. L. and Sullivan, G. R. (1988) Generic model control (GMC). Comput. Chem. Engng 12, 573-580.

Lewis, F. L. (1992) Applied Optimal Control and Estimation. Prentice-Hall, Englewood Cliffs, NJ, U.S.A.

Meditch, J. S. and Hostetter, G. H. (1974) Observers for systems with unknown and inaccessible inputs. Int. J. Control 19, 473-480.

Misawa, E. A. (1988) Nonlinear state estimation using sliding observers. Ph.D. thesis, Massachusetts Institute of Technology, Cambridge, U.S.A.

Misawa, E. A. and Hedrick, J. K. (1989) Nonlinear observers - a state-of-the-art survey. ASME $J$. Dyn. System Measurement Control 111, 344-352.

Morari, M. and Stephanopoulos, G. (1980) Part II: structural aspects and the synthesis of alternative feasible control schemes. A.I.Ch.E. J. 26, 232-246.

Slotine, J.-J. E., Hedrick, J. K. and Misawa, E. A. (1987) On sliding observers for nonlinear systems. ASME J. Dyn. System Measurement Control 109, 245-252.

Slotine, J.-J. E. and Li, W. (1991) Applied Nonlinear Control. Prentice-Hall, Inc., Englewood Cliffs, NJ, U.S.A.

Slotine, J.-J. E. and Sastry, S. S. (1983) Tracking control of nonlinear systems using sliding surfaces with application to robot manipulators. Int. J. Control 38, 465-492.

Stewart, G. W. and Sun, J. G. (1993) Matrix Perturbation Theory. Academic Press, San Diego, U.S.A.

Vidyasagar, M. (1993) Nonlinear Systems Analysis. Prentice-Hall, Englewood Cliffs, NJ, U.S.A.

\section{APPENDIX A: DERIVATIONS FOR EQ. (28)}

From eq. (17), we have

$$
\dot{\tilde{\mathbf{x}}}_{r}=H \tilde{\mathbf{x}}_{r}+\boldsymbol{\delta}_{r} .
$$

Thus, we can solve that

$$
\tilde{\mathbf{x}}_{r}=\mathrm{e}^{H} \tilde{\mathbf{x}}_{r}(0)+\int_{0}^{t} \mathrm{e}^{H(t-\tau)} \delta_{r} \mathrm{~d} \tau .
$$

Let $\left\|\tilde{\mathbf{x}}_{r}(0)\right\| \leqslant a$; wc havc

$$
\begin{aligned}
\left\|\tilde{\mathbf{x}}_{r}\right\| & \leqslant a\left\|\mathrm{e}^{H t}\right\|+\int_{0}^{t}\left\|\mathrm{e}^{H(t-\tau)}\right\|\left\|\boldsymbol{\delta}_{r}\right\| \mathrm{d} \tau \\
& \leqslant a \mathrm{e}^{-\lambda_{m^{t}}}+\int_{0}^{t} \mathrm{e}^{-\lambda_{m}(t-\tau)}\left\|\boldsymbol{\delta}_{r}\right\| \mathrm{d} \tau
\end{aligned}
$$

where $-\lambda_{m}\left(\lambda_{m}>0\right)$ is the greatest eigenvalue of $H(\hat{\mathbf{x}})$. From eqs (20), (22) and (25), it is known that

$$
\boldsymbol{\delta}_{\boldsymbol{r}} \equiv \delta \mathbf{f}_{r}-\frac{\delta f_{1}}{k_{1}} \mathbf{k}_{r} .
$$


Then it can be derived that

$$
\left\|\boldsymbol{\delta}_{r}\right\| \leqslant\left\|\delta \mathbf{f}_{r}\right\|+\frac{\left|\delta f_{1}\right|}{k_{1}}\left\|\mathbf{k}_{r}\right\| \leqslant\|\delta \mathbf{f}\|\left(1+\frac{\left\|\mathbf{k}_{r}\right\|}{k_{1}}\right) .
$$

Therefore, if there exist $b, N$ and $\varepsilon$ such that

$$
\|\partial \mathbf{f}\| \leqslant\left(b+N \mathrm{e}^{-c r}\right) /\left(1+\left\|\mathbf{k}_{\mathbf{r}}\right\| / k_{1}\right)
$$

where $b, N$ and $\varepsilon$ are all positive constants and $\varepsilon<\lambda_{m}$, we shall obtain

$\left\|\tilde{\mathbf{x}}_{\mathbf{r}}\right\| \leqslant a \mathrm{e}^{-\lambda_{m^{t}}}+\mathrm{e}^{-\lambda_{m^{t}}} \int_{0}^{t} \mathrm{e}^{\lambda_{m^{2}}}\left[b+N \mathrm{e}^{-\varepsilon t}\right] \mathrm{d} t$

$$
\begin{aligned}
& =a \mathrm{e}^{-\lambda_{m} t}+\frac{b}{\lambda_{m}}\left(1-\mathrm{e}^{-\lambda_{m} t}\right)+\frac{N}{\lambda_{m}-\varepsilon}\left(\mathrm{e}^{-\varepsilon t}-\mathrm{e}^{-\lambda_{m} t}\right) \\
& =a \mathrm{e}^{-\lambda_{m} t}+\frac{b}{\lambda_{m}}\left(1-\mathrm{e}^{-\lambda_{m} t}\right)+\frac{N}{\lambda_{m}-\varepsilon} \mathrm{e}^{-\varepsilon 1}\left[1-\mathrm{e}^{-\left(\lambda_{m}-\varepsilon\right) t}\right] \\
& \leqslant a \mathrm{e}^{-\lambda_{m} t}+\frac{b}{\lambda_{m}}+\frac{N}{\lambda_{m}-\varepsilon} \mathrm{e}^{-\varepsilon t} .
\end{aligned}
$$

Hence, it is proved that $\left\|\tilde{\mathbf{x}}_{r}\right\|$ is bounded. 